\title{
Ordinal and Probabilistic Representations of Acceptance
}

\author{
Didier Dubois \\ Helene Fargier \\ Henri Prade \\ Institut de Recherche en Informatique de Toulouse \\ Université Paul Sabatier 118 route de Narbonne \\ 31062 Toulouse Cedex 4 France
}

DUBOIS@IRIT.FR

FARGIER@IRIT.FR

PRADE@IRIT.FR

\begin{abstract}
An accepted belief is a proposition considered likely enough by an agent, to be inferred from as if it were true. This paper bridges the gap between probabilistic and logical representations of accepted beliefs. To this end, natural properties of relations on propositions, describing relative strength of belief are augmented with some conditions ensuring that accepted beliefs form a deductively closed set. This requirement turns out to be very restrictive. In particular, it is shown that the sets of accepted belief of an agent can always be derived from a family of possibility rankings of states. An agent accepts a proposition in a given context if this proposition is considered more possible than its negation in this context, for all possibility rankings in the family. These results are closely connected to the non-monotonic 'preferential' inference system of Kraus, Lehmann and Magidor and the so-called plausibility functions of Friedman and Halpern. The extent to which probability theory is compatible with acceptance relations is laid bare. A solution to the lottery paradox, which is considered as a major impediment to the use of non-monotonic inference is proposed using a special kind of probabilities (called lexicographic, or big-stepped). The setting of acceptance relations also proposes another way of approaching the theory of belief change after the works of Gärdenfors and colleagues. Our view considers the acceptance relation as a primitive object from which belief sets are derived in various contexts.
\end{abstract}

\section{Introduction}

There is an old controversy in the framework of Artificial Intelligence (AI) between probabilistic and other numerical representations of uncertainty on the one hand, and the symbolic setting of logical reasoning methods on the other hand. Namely, the AI tradition maintains that knowledge representation and reasoning should rely on logic (Minker, 2000), while the probabilistic tradition sticks to numerical representations of belief. The emergence and relative success of some numerical reasoning formalisms based on probability (especially Bayesian networks) or fuzzy sets has nevertheless led AI to accept numerical representations as complementary to symbolic ones. However the basic issue underlying the controversy, that is to say, whether or not the two forms of reasoning (logical and numerical) are at all compatible at the formal level, has not been widely addressed. Namely suppose the beliefs of an agent are represented simultaneously by a measure of confidence (such as a probability function for instance) on a set of states, and by a logical belief base. To what extent can the two representations be consistent with each other?

In order for this question to make sense, we need a formal connection between the two representations and especially, we must extract so-called accepted beliefs from the confidence measure so as to feed a belief base. The question of accepted beliefs has been discussed in 
epistemology for a long time. It is not widely agreed how to define an accepted belief and several schools of thought exist. Swain (1970) provides basic material on this topic. Usually, accepted beliefs are understood as propositions whose degrees of belief are high enough to be considered as true, and they form a deductively closed set. However this view is challenged by probabilists. One proposal of this paper is a definition of accepted belief according to a confidence relation, a relation comparing propositions in terms of their relative credibility for an agent : a minimal requirement for a proposition $p$ to be a belief is that the agent believes $p$ more than its negation. This is the weakest definition one may think of. The specific requirement for $p$ to be an accepted belief is that the agent is ready to infer from $p$ in a classical sense, as if $p$ were true. Then, two questions are worth investigating :

1) Under what conditions can the set of beliefs according to a confidence measure coincide with the deductive closure of a logical belief base?

2) Upon arrival of new information, can the set of beliefs obtained from the measure of confidence after conditioning, and the revised belief base remain in agreement as well?

Citing Cohen (1977), the paper explores the common ground between "the probable and the provable". Philosophers like Kyburg (1961) considered this question in the past. He proposed the lottery paradox as evidence that probability theory is inconsistent with deductive closure and the notion of accepted belief. More recently, it has been pointed out that the lottery paradox also questions the credibility of the non-monotonic reasoning school (Kyburg, 1988; Poole, 1991). This paper casts the problem of the reasonableness of deductive closure for accepted beliefs in a more general ordinal setting, using relations for comparing events in terms of relative likelihood, certainty, plausibility and the like, in connection with the AGM theory of belief revision, after Alchourron et al. (1985) and Gärdenfors (1988). Such relations can be induced by numerical representations of belief such as probability (Fishburn, 1986), possibility (Lewis, 1973; Dubois, 1986), Shafer's belief and plausibility functions (Wong et al., 1991).

This paper shows that the assumption that beliefs extracted from a confidence relation form a deductively closed set severely restricts the type of comparative belief structure that can be envisaged for reasoning under uncertainty. Actually, the range of compatibility between deductive closure and uncertain reasoning basically reduces to possibility theory. It also precisely covers the kind of non-monotonic inference systems called "System P" (Kraus, Lehmann \& Magidor, 1990), rational closure (Lehmann \& Magidor, 1992). Our work is thus closely related to the works of Friedman and Halpern (1996) who describe the semantics of non-monotonic inference in terms of plausibility functions valued on a partially ordered set. This approach has close connections with various works carried out more or less independently by philosophers like Ernest Adams (1975), David Lewis (1973), in the seventies, and Peter Gärdenfors (1988) and colleagues in the eighties, as well as several AI researchers, such as Yoav Shoham (1988), Daniel Lehmann and colleagues (1990, 1992), Judea Pearl (1990), Joe Halpern (1997), Maryanne Williams (1994), Hans Rott (2001) and others.

In this paper, we also provide a direct proof that confidence relations compatible with deductive closure can be substituted by a family of possibility relations characterizing the same set of accepted beliefs. Moreover, we also demonstrate some compatibility between the notion of logical closure and probability theory and suggest a solution to the lottery paradox. The paper is a continuation of previous work: Dubois and Prade (1995a) proposed a definition of accepted belief. Later, Dubois Fargier and Prade (1998) presented some results on acceptance relations and their numerical counterparts. 


\section{REPRESENTATIONS OF ACCEPTANCE}

The paper is organized as follows : Section 2 recalls the lottery paradox as a motivation for this research. It introduces comparative belief structures and suggests postulates governing the notion of accepted beliefs, and their revision in the face of new information by means of conditioning. Some examples of acceptance relations are discussed, especially the so-called possibility relations, each being characterized by a complete preordering of states. Comparative probabilities that are acceptance relations are laid bare and shown to be a very restricted class, which can be represented by big-stepped (lexicographic) probabilities. Section 3 presents the main representation results for acceptance relations. We show that the useful part of any acceptance relation, that is, the part from which accepted beliefs are derived, can be represented by a family of possibility relations. Section 4 relates these results to existing works in non-monotonic inference and probabilistic reasoning. It also revisits belief revision theory in the light of acceptance relations. Lastly, we show that big-stepped probabilities are immune to the lottery paradox, and suggest a range of situations where reasoning deductively with accepted beliefs makes sense.

\section{Accepted Beliefs}

Consider an agent who has to reason about the current state of the world. The body of information possessed by the agent is supposed to contain (at least) three distinct types of items: observations pertaining to the current situation, generic knowledge about similar situations, and beliefs regarding the non-directly observable features of the current situation. In logical approaches (Minker, 2000), observations and generic knowledge are encoded in some logic-based language. In probabilistic approaches, generic knowledge is modeled by a probability distribution on the set of possible situations, and often encoded as a Bayes net, for instance. Observations result in the partial instantiation of some variables. Plausible reasoning consists in inferring beliefs from (contingent) observations, and generic (background) knowledge, valid across situations. In logical approaches this is achieved via logical (generally, non-monotonic) deduction. In probability theory, it consists in computing conditional probabilities of relevant propositions, where the conditioning event gathers the available observations (De Finetti, 1974). Observations are supposed to be reliable and non-conflicting, while computed beliefs are on the contrary taken for granted, hence brittle. There is a strong similarity between the logical and the probabilistic approaches to plausible reasoning. Actually, it seems that the ultimate aim of symbolic AI in plausible reasoning is to perform a counterpart to probabilistic inference without probabilities (Dubois \& Prade, 1994).

\subsection{The Lottery Paradox}

The main difference between logic-based and probabilistic approaches to plausible reasoning is that in logic-based approaches, accepted beliefs form a deductively closed set of propositions, while the aim of probabilistic reasoning is to assign degrees of belief to propositions of interest.

In particular, the classical logic approach to belief representation considers a set $\boldsymbol{B}$ of logical formulas $\phi, \psi, \ldots$ forming a set of explicit beliefs of an agent, from which it is possible to infer implicit beliefs. The set $\operatorname{cons}(\boldsymbol{B})$ of consequences of $\boldsymbol{B}$ is the set of "rationally" accepted beliefs also called a belief set $\boldsymbol{K}$ (Gärdenfors, 1988).

In numerical representations of belief, propositions are modeled by subsets of a (here, finite) set $S$. Elements of $S$ are called "states". They encode descriptions of possible situations, states of affairs, etc. Subsets $A, B, C$, of states are called events. The finiteness assumption is here made 
for the sake of simplicity, and is natural when the description of situations is achieved by means of a formal language such as propositional logic. Then, $S$ is the set of interpretations of the language. It comes down to understanding propositions as ultimately true or false. Here we consider a syntax-free environment, from a logical point of view. In particular, a proposition is understood as the subset of states where it is true, so events coincide with propositions. In order to relate belief sets and numerical plausible reasoning, we identify events to sets $A=[\phi]$ of models of logical formulas $\phi$ and use the event notation, $A, B, C$. $A \cap B, A \cup B$ and $\neg A$ denote the intersection of $A$ and $B$, the union of $A$ and $B$ and the complementation of $A$, respectively.

The question is now how to model an accepted belief in the sense of a probability measure $P$. The most natural view is to set a threshold $\alpha$ and assume that an agent accepts a proposition $A$ whenever $P(A)>\alpha$; the book by Swain (1970) contains a precise account of variants of this proposal. Obviously one must choose $\alpha=1 / 2$, because a minimal condition for $A$ to be believed is that $P(A)>P(\neg A)$. Consider the set $\boldsymbol{A}_{P}=\{A, P(A)>\alpha\}$ of accepted beliefs. First it is easy to see that in general, this set is not deductively closed, since whatever the choice of $\alpha$ in $[0.5,1)$, one may have $A \in A_{P}, B \in A_{P}$, but their conjunction $A \cap B \notin A_{P}$, because more often than not, $P(A \cap B)<\alpha$. So the set $\boldsymbol{A}_{P}$ is generally not a closed belief set $\boldsymbol{K}$. Even worse, $\boldsymbol{A}_{P}$ is sometimes logically inconsistent. This critique of conjunctive closure for accepted beliefs was made a long time ago by Kyburg $(1961,1970)$. Scholars in probability theory, far from considering these facts as invalidating the probabilistic approach to plausible reasoning, have on the contrary questioned the relevance of logic-based approaches on this basis. The main objection is captured by the lottery paradox, that was proposed as a counterexample to the use of classical deduction on accepted beliefs and to non-monotonic reasoning at large (Kyburg, 1988; Poole, 1991).

Suppose $n>1,000,000$ lottery tickets are sold and there is one winner ticket. So the probability $P$ (player $i$ loses) $>0.99$ 999. That is, one should believe that player $i$ loses and $\boldsymbol{A}_{P}$ contains the propositions "player $i$ loses" for all $i$. If beliefs are deductively closed, the agent should conclude that all players lose. However, since there is one winning ticket, Prob(all players lose) $=0$, for any value of $n$. That is, the proposition "one player wins" lies in $\boldsymbol{A}_{P}$. Hence, $\boldsymbol{A}_{P}$ is inconsistent, and accepted beliefs cannot match with high probability events, whatever "high" means. Deductive reasoning with accepted beliefs looks counterintuitive. This example seems to kill any attempt to exploit logical approaches in the computation of accepted beliefs. Yet, our claim in this paper is that, contrary to what the lottery paradox would suggest, there does exist some consistency between uncertainty theories and symbolic approaches to plausible reasoning. However,

1)Requesting that the set of accepted beliefs in the sense of some uncertainty theory be always deductively closed severely restricts the choice of such an uncertainty theory.

2) There exists some subset of probability measures for which the set $\boldsymbol{A}_{P}=\{A, P(A)>0.5\}$ of accepted beliefs is deductively closed. But they are probability measures for which some states of the world appear to be much more probable than others, in any context. Examples like the lottery paradox fall out of this range as they presuppose a uniformly distributed probability.

Rather than addressing the problem for probability theory setting only, we first try to cast it in a general ordinal setting, where the background knowledge of the agent is represented by a general relation between events - that we call a "confidence relation", having minimal rationality properties. This framework is useful to isolate uncertainty theories that are consistent with deductive closure. 


\section{REPRESENTATIONS OF ACCEPTANCE}

\subsection{Confidence Relations}

The knowledge of an agent about the normal state of the world is supposed to be modeled by a relation $\geq_{L}$ among events that are subsets of a finite set $S$. Stating $A \geq_{L} B$ means "the agent has at least as much confidence in event $A$ as in event $B$ ", and $L$ stands for "likelihood". The pair $\left(2^{S}, \geq_{L}\right)$ is called a comparative belief structure and relation $\geq_{L}$ is called a confidence relation, under conditions to be prescribed below. From this confidence relation, other associated relations are defined in the usual way (Roubens \& Vincke, 1985) :

- $A>_{L} B$ if and only if $\left(A \geq_{L} B\right)$ and not $\left(B \geq_{L} A\right)$ (strict preference);

- $A \#_{L} B$ if and only if neither $\left(A \geq_{L} B\right)$ nor $\left(B \geq_{L} A\right)$ (incomparability);

- $A \equiv_{L} B$ if and only if $\left(A \geq_{L} B\right)$ and $\left(B \geq_{L} A\right)$ (indifference).

Let us set the minimal properties required for a confidence relation. First, it is hard to imagine a situation where the agent's confidence in $A$ is strictly higher than his confidence in $B$, and this agent's confidence in $B$ is also strictly higher than his confidence in $C$, while $A$ is not more likely than $C$. The strict part $>_{L}$ of a confidence relation $z_{L}$ is thus naturally assumed to be transitive, i.e. the relation $\geq_{L}$ is supposed to be quasi-transitive :

Quasi-transitivity $(\mathrm{QT}): A>_{L} B$ and $B>_{L} C$ imply $A>_{L} C$

But the transitivity of the indifference relation it induces is not required. For instance, indifference between two events may be due to imperfect discernment about confidence levels and cannot always be chained. The incomparability relation is of course not transitive.

Moreover, if $A \subseteq B$, then $A$ implies $B$. So it should be that $A$ and $B$ are comparable, and that the agent's confidence in $A$ cannot be strictly greater than in $B$. It reads:

\section{Monotonicity with respect to inclusion (MI): if $A \subseteq B$ then $B \geq_{L} A$.}

This property of monotonicity forbids situations where a proposition would be more believed than another it semantically entails. But it is not sufficient to ensure that the strict part of $z_{L}$ is coherent with classical deduction. That is why the orderly axiom of Halpern (1997) must be added.

Orderly axiom $(\mathrm{O}):$ if $A \subseteq A^{\prime}, B^{\prime} \subseteq B$, and $A>_{L} B$, then $A^{\prime}>_{L} B^{\prime}$

It means that if $A$ is more likely than $B$, then if $A^{\prime}$ is a consequence of $A$ while $B^{\prime}$ implies $B$, it should be that $A^{\prime}$ is more likely than $B^{\prime \prime}$. It should be emphasized that axiom $\mathrm{O}$ does not imply MI, because due to incompleteness of $\geq_{L}$ it may occur that $A \subset B$ and $A \#_{L} B$. Symmetrically, MI does not imply $\mathrm{O}$ because $A>_{L} C$ does not follow from $A>_{L} B$ and $B \geq_{L} C$. For instance, if the confidence relation $\geq_{L}$ is quasi-transitive but not transitive, we may have that $A>_{L} B, B \geq_{L} C$ and $A \#_{L} C$. The reader will find in Appendix 1 a detailed study on the relationships between axioms $\mathrm{O}$ and $\mathrm{MI}$, with respect to the assumption of quasi-transitivity, full transitivity and completeness. Hence the following definition, which uses minimal requirements for a confidence relation :

\footnotetext{
${ }^{1}$ Axiom $\mathrm{O}$ applied to $\geq_{L}$ also ensures the same sound behaviour of the strict confidence relation. Indeed, if $\geq_{L}$ satisfies $O$, it is easy to show that relation $>_{L}$ also satisfies $\mathrm{O}$.
} 
Definition 1. A confidence relation is a relation on $2^{S}$, satisfying $O, Q T$, and MI.

The following properties of a confidence relation are obvious consequences of this definition :

Reflexivity of $\geq_{L}$ and thus of $\equiv_{L}(R): \quad A \geq_{L} A$ (and thus $A \equiv_{L} A$ ) hold.

Irreflexivity of $>_{L}(I R): A>_{L} A$ does not hold $\left(A>_{L} A\right.$ makes no sense).

Partial belief is often represented by numerical functions such as probability measures, which map events to a numerical scale.

Definition 2. A confidence function $f$ is a [0,1]-valued set-function such that $f(S)=1, f(\varnothing)$ $=0$ and, if $A \subseteq B$, then $f(B) \leq f(A)$.

A probability measure is a confidence function ${ }^{2}$. A confidence function $f$ obviously defines a confidence relation $\geq_{f}$ as follows :

$$
A \geq_{f} B \text { if and only if } f(A) \geq f(B) .
$$

It is easy to check that $\geq_{f}$ satisfies $M I, O, Q T$, and the two following ones:

Transitivity (T): If $A \geq_{f} B, B \geq_{f} C$ then $A \geq_{f} C$, for all $A, B, C$;

Completeness (CL): either $A \geq_{f} B$ or $B \geq_{f} A$ for all $A, B$;

The converse also holds : it is easy to build a confidence function by mapping a complete and transitive confidence preorder to the unit interval. A transitive confidence relation will be called a confidence preorder - it is reflexive and transitive, but may be partial. It should be noticed that axiom $O$ can be recovered from $M I$ if full transitivity of $z_{L}$ is assumed (see Appendix 1). Axioms $O$ and $M I$ are actually equivalent when the confidence preorder is also complete. A complete confidence preorder is thus a complete preorder on events satisfying MI. In our more general setting, these properties are not required a priori. For instance, the completeness of $z_{L}$, which is equivalent to the emptiness of the incomparability relation, is not compulsory : indeed, when an agent describes uncertain knowledge, only part of the relation may be available. In this paper, we leave room for such incomplete relations and we interpret the incomparability $A \#_{L} B$ as a lack of knowledge regarding the relative likelihood of events $A$ and $B$.

\subsection{Noticeable Confidence Relations}

Some well-known confidence relations satisfy a stability property with respect to adding or deleting elements common to any two sets (Fishburn, 1986):

\footnotetext{
${ }^{2}$ In the literature, confidence functions are called capacities (this name was created in the context of electrical engineering by the mathematician Choquet (1953) or fuzzy measures (coined by Sugeno (1974) in connection with fuzzy set theory).
} 


\section{REPRESENTATIONS OF ACCEPTANCE}

Definition 3 (preadditivity). Let $A, B$ and $C$ be any three events such that $A \cap(B \cup C)=$ $\varnothing$. A confidence relation $\geq_{L}$ is preadditive whenever $A \cup B \geq_{L} A \cup C$ if and only if $B \geq_{L} C$.

The idea is that set $A$ should not affect the relative likelihood of $A \cup B$ and $A \cup C$ since it is common to both events. Denoting $\geq_{L}^{T}$ the dual of $\geq_{L}$, such that $A \geq_{L}{ }^{T} B$ if and only if $\neg B \geq_{L} \neg A$, it can be checked that preadditive relations are self-dual, i.e., $A \geq_{L} B$ if and only if $\neg B \geq_{L} \neg A$. For instance, comparative probabilities (Fishburn, 1986) are preadditive confidence preorders. Probability functions induce such type of relations, but not all such relations can be represented by probability functions (Kraft et al, 1959).

Lewis (1973) introduced another confidence relation in the setting of a modal logic of counterfactuals (it was independently rediscovered by Dubois, 1986) : the possibility relation.

Definition 4. A set-relation $\geq_{\Pi}$ is a possibility relation if and only if it is complete, transitive, non trivial $\left(S>_{\Pi} \varnothing\right)$ and satisfies the following axioms

$\bullet \forall \subseteq S, A \geq_{\Pi} \varnothing ;$

- $\forall A, B, C, B \geq_{\Pi} C$ implies $A \cup B \geq_{\Pi} A \cup C$ (unrestricted union stability).

$B \geq_{\Pi} C$ reads, " $B$ is at least as possible (that is, plausible for the agent) as $C$ ", for reasons that will become clear below. Such relations are neither preadditive nor self-dual. The dual setrelation defined by $A \geq_{N} B$ if and only if $\neg B \geq_{\Pi} \neg A$ is called a necessity relation. It can be independently defined as follows:

Definition 5. A set-relation $\geq_{N}$ is a necessity relation if and only if it is complete, transitive, non trivial $\left(S>_{N} \varnothing\right)$ and satisfies the following axioms:

$\bullet A \subseteq S, A \geq_{N} \varnothing$;

$\cdot \forall A, B, C, B \geq_{N} C$ implies $A \cap B \geq_{N} A \cap C$ (unrestricted intersection stability).

$B \geq_{\mathrm{N}} C$ reads, " $B$ is at least as necessary (that is, certain for the agent) as $C$ ". Besides, the less plausible is $\neg A$, the more certain is $A$, so that $A>_{N} B$ means that $A$ is more certain than $B$. The duality between possibility and necessity relations is a translation of the duality between possibility and necessity modalities in modal logic (Fariñas del Cerro \& Herzig, 1991).

Possibility and necessity relations are fully characterized by a complete preorder $\geq_{\pi}$ of states in $S$, defined by:

$$
s \geq_{\pi} s^{\prime} \quad \text { if and only if } \quad\{s\} \geq_{\Pi}\left\{s^{\prime}\right\} \quad \text { if and only if } \quad S\left|\left\{s^{\prime}\right\} \geq_{N} S\right|\left\{s^{\prime}\right.
$$

The relation $\mathrm{s} \geq_{\pi} \mathrm{s}^{\prime}$ means that $\mathrm{s}^{\prime}$ is at least as plausible as $\mathrm{s}$. Event $\mathrm{A}$ is plausible to the extent to which its most normal realization is plausible : the original possibility (and thus necessity) relations for non empty sets can be recovered from $\left(S, \geq_{\pi}\right)$ as follows:

$$
\begin{aligned}
& \forall A \neq \varnothing, \forall B \neq \varnothing, A \geq_{\Pi} B \text { if and only if } \exists s \in A, \forall s \in B, s \geq_{\pi} s^{\prime} \\
& \forall A \neq \varnothing, \forall B \neq \varnothing, A \geq_{N} B \text { if and only if } \exists s \notin B, \forall s \notin A, s \geq_{\pi} s^{\prime}
\end{aligned}
$$


The exact and only set-functions that represent possibility relations are possibility measures (Dubois, 1986). A possibility measure $\Pi$ is a confidence function satisfying $\Pi(A \cup B)=$ $\max (\Pi(A), \Pi(B))$ (Zadeh, 1978; Dubois \& Prade, 1998a). Necessity measures are defined dually from possibility measures as $N(A)=1-\Pi(\neg A)$. They are the exact and only confidence functions that represent necessity orderings.

\subsection{Accepted Beliefs and Their Revision in the Confidence Relation Setting}

A confidence relation $\geq_{L}$ is supposed to exist, that represents the body of generic knowledge of an agent: it describes what this agent believes more or less strongly to be usually true for the problem under consideration. The weakest and most natural definition of what it means for a proposition $A$ to be a belief for an agent in the sense of a confidence relation $\geq_{L}$ is that the agent is more confident in $A$ than in its negation (modeled by the complement $\neg A$ of the set $A$ ):

Definition 6. Let $\geq_{L}$ be a confidence relation. $A$ is a belief for $\geq_{L}$ if and only $A>_{L} \neg A$

An accepted belief should first be a belief. In Subsection 2.1, a proposition is tentatively accepted if its probability is high enough. As pointed out earlier, the smallest acceptance threshold is 0.5 , and $P(A)>0.5$ is equivalent to $P(A)>P(\neg A)$. The latter condition makes sense in the ordinal setting and reads $A>_{L} \neg A$. Another definition of beliefs would use some prescribed proposition $T>_{L} \neg T$ as a threshold and consider as a belief any other proposition $A \geq_{L} T$. However $A>_{L} \neg A$ should also hold for such propositions, because there would be no reason to believe $A$ rather than its negation otherwise. Ours is the weakest notion of belief one may think of. And the results found in this paper also apply to alternative proposals.

Definition $7:$ Let $\geq_{L}$ be a confidence relation on $S$. The set of beliefs according to $\geq_{L}$, denoted $\boldsymbol{A}_{L}$, is defined by: $\boldsymbol{A}_{L}=\left\{A: A>_{L} \neg A\right\}$.

It must be clear that the confidence relation $\geq_{L}$ and its belief set $\boldsymbol{A}_{\mathrm{L}}$ are not equivalent notions. The belief set is always deduced from the confidence relation, but neither the original confidence relation nor even its strict part can be re-built from this belief set alone. By assumption, the relation $\geq_{L}$ represents a body of generic knowledge. In contrast, $\boldsymbol{A}_{\mathrm{L}}$ represents the agent's beliefs about the current situation.

An accepted belief is a proposition an agent is ready to consider as being true, that is, the agent is ready to infer classically from it. This view of acceptance is shared by philosophers like Cohen (1989):

"...to accept that $p$ is to have or adopt a policy of deeming, positing or postulating that $p-$ that is, of going along with this proposition... as a premiss in some or all contexts for one's own or others 'proofs, argumentations, deliberations etc."

while "belief that $p$... is a disposition to feel it true that $p "$.

Confidence relations that model acceptance thus model a very strong kind of belief, one such that an agent is ready to take for granted in the course of deliberations. In order to remain compatible with logic-based representations, a set of accepted beliefs $\boldsymbol{A}_{\mathrm{L}}$ must thus be deductively closed. It requires that any consequence of an accepted belief be an accepted belief and that the conjunction of two accepted beliefs be an accepted belief as well (Dubois \& Prade, 1995b). 


\section{REPRESENTATIONS OF ACCEPTANCE}

Insofar as accepted propositions are built from a special kind of confidence relation, it leads to the following conditions that such confidence relations should satisfy :

Entailment stability (ES) : if $A \subseteq B$ and $A>_{L} \neg A$ then $B>_{L} \neg B$

Conjunction stability (CS): if $A>_{L} \neg A$ and $B>_{L} \neg B$ then $A \cap B>_{L} \neg A \cup \neg B$.

Axiom CS is clearly the most controversial one (Kyburg, 1970), but is nevertheless adopted by many scholars, like Hempel, Hintikka, Lehrer, Levi, etc. (see Swain , 1970, for a full bibliography and discussions). Axioms ES and CS ensure that a belief set $\boldsymbol{A}_{\mathrm{L}}$ never contains $\varnothing$, hence is always logically consistent, by construction (indeed, if the conjunction of all accepted belief is $\varnothing$, then we would have, under CS, $\varnothing>_{L} S$, which contradicts $M I$ ).

Property $E S$ holds for any confidence relation due to the orderly property $O$. But the deductive closure of the set of accepted beliefs is not guaranteed for any confidence relation. In the probabilistic case, CS is generally not satisfied and $\boldsymbol{A}_{\mathrm{P}}$ may be a logically inconsistent knowledge base: probabilistic beliefs are generally not strong enough to be used in deductive inference processes but, as we shall see later, some probability functions may model beliefs strong enough to be accepted.

While the set of accepted beliefs according to a probability relation is not closed under conjunction as already pointed out, the set of beliefs induced by a possibility or a necessity relation is a deductively closed set. Namely assume that $A>_{\Pi} \neg A$, and $B>_{\Pi} \neg B$. Using the definitions of section 2.3, it is clear that there exists an element $s_{A} \in A$, such that $s_{A} \geq_{\pi} s, \forall \mathrm{s} \in A$. Moreover, $s_{A}>_{\pi} s, \forall \mathrm{s} \notin$ A. Hence $s_{A} \geq_{\pi} s, \forall \mathrm{s} \in S$. So, since $B>_{\Pi} \neg B, s_{A} \in B$, and $s_{A}>_{\pi} s, \forall \mathrm{s} \notin$ B. Hence, $s_{A} \in A \cap B$, and $s_{A}>_{\pi} s, \forall \mathrm{s} \notin \mathrm{A}, \mathrm{s} \notin \mathrm{B}$. Hence $A \cap B>_{\Pi} \neg(A \cup B)$.

This result is also easily guessed by referring to Gärdenfors theory of revision. Indeed, the AGM belief revision theory assumes that a belief set is deductively closed. A number of axioms are proposed which a belief revision operator should satisfy. These axioms lead to the underlying existence of a confidence relation called an epistemic entrenchment relation. It was pointed out (Dubois \& Prade, 1991) ${ }^{3}$ that this underlying epistemic entrenchment relation is a necessity relation $\geq_{N}$ such that $S>_{N} A, \forall A \neq \varnothing$, i.e. no contingent proposition is as sure as the sure event. The belief set is thus $\boldsymbol{K}=\left\{A, A>_{N} \neg A\right\}$. Note that $A>_{L} \neg A$ is equivalent to the dual $A>_{L}^{T} \neg A$. Also note that in the case of necessity relations, $A>_{N} \neg A$ is equivalent to $A>_{N} \varnothing$, since it cannot be that $A>_{N} \varnothing$ and $\neg A>_{N} \varnothing$ for any event $A$ at the same time. So, $\boldsymbol{K}=\left\{A, A>_{\Pi} \neg A\right\}$. This not only shows that the set of beliefs induced by a possibility relation is deductively closed, but also that our definition of accepted belief also generalizes the definition of belief that is implicit in Gärdenfors theory of revision.

Now, due to incomplete knowledge, an accepted belief in $\boldsymbol{A}_{\mathrm{L}}$ is only tentatively accepted and may be questioned by the arrival of new information: the arrival of observations made by the agent leads to a revision of the set of accepted beliefs. Consider that the information received by the agent about the current situation summarized under the form of a proposition $C$.

In belief revision theory, rationality axioms specify how a revision operator should behave, especially when the initial belief set $\boldsymbol{K}$ is contradictory with the input information C (Gärdenfors, 1988 ; see also Section 4.2). In this paper, rather than following this axiomatic path, we shall use the notion of conditioning for the integration of new evidence pertaining to the current situation.

\footnotetext{
${ }^{3}$ Grove (1988) has also described belief revision operations in terms of a relation dual to an epistemic entrenchment relation, hence a possibility relation.
} 


\section{DUBOIS, FARGIER, \& PRADE}

And we shall require that the set of accepted beliefs after conditioning should remain a belief set. A new belief set, $A_{\mathrm{L}}(C)$ can then be defined from $C$ and $\geq_{L}$. According to our requirements, $A_{\mathrm{L}}(C)$ must be deductively closed.

We take it for granted that conditioning a confidence relation on a set $\mathrm{C}$ representing a piece of evidence about the current situation comes down to restricting the confidence relation to subsets of C. This is the idea of focusing on a reference class, which was suggested earlier as one interpretation of conditioning (Dubois, Moral \& Prade, 1998). This is the natural counterpart to Bayesian conditioning for confidence relations. Indeed, in numerical uncertainty theories, the axioms of conditioning have been studied using the following equation (Cox, 1961): $f(A \mid C)$ is a solution to the equality $f(A \cap C)=f(A \mid C) * f(C)$ where $*$ is continuous and strictly monotonic in both places. Cox and followers (e.g. Paris, 1994) prove that the only possible solution is $*=$ product - see (Halpern, 1999) for a discussion. Hence $f(A \mid C) \geq f(\neg A \mid C)$ if and only if $f(A \cap C)$ $\geq f(A \cap C){ }^{4}$

Definition 8: Let $\geq_{L}$ be a confidence relation. The confidence in $A$ is said to be at least as high as the confidence in $B$ in the context $C \subseteq S$ if and only if $A \cap C \geq_{L} B \cap C$. The induced set of beliefs in context $C$ is $: \boldsymbol{A}_{L}(C)=\left\{A: A \cap C>_{L} \neg A \cap C\right\}$.

Clearly, $\boldsymbol{A}_{\mathrm{L}}=\boldsymbol{A}_{\mathrm{L}}(S)$. Going from $\boldsymbol{A}_{\mathrm{L}}$ to $\boldsymbol{A}_{\mathrm{L}}(C)$ is a natural way of revising a set of currently held beliefs $\boldsymbol{A}_{\mathrm{L}}$, on the basis of a confidence relation and a new information item $C$ about the current situation. Note that revising the confidence relation itself is another problem not dealt with here. In this paper we only consider the change of current beliefs about a particular situation when prior generic knowledge is encoded by the confidence relation, which only results from focusing the generic knowledge on the proper subclass of states pertaining to available observations.

Now, the requirement that sets of accepted beliefs $A_{\mathrm{L}}(C)$ be deductively closed means that, if $A$ is believed in context $C$ and $B$ is entailed by $A$, then $B$ should be believed in context $C$ (Conditional entailment stability) and that, moreover, if $A$ and $B$ are both believed in context $C$, then, so should be the conjunction $A \cap \mathrm{B}$ (Conditional Conjunctive Stability). These properties enforce conditional versions of the ES and CS conditions for the confidence relations (Dubois \& Prade, 1995a):

Conditional Entailment Stability (CES):

$$
\text { If } A \subseteq B \text { and } C \cap A>_{L} C \cap \neg A \text { then } C \cap B>_{L} C \cap \neg B \text {; }
$$

Conditional Conjunction Stability (CCS) :

$$
\text { If } C \cap A>_{L} C \cap \neg A \text { and } C \cap B>_{L} C \cap \neg B \text { then } C \cap A \cap B>_{L} C \cap(\neg A \cup \neg B) \text {. }
$$

These are necessary and sufficient conditions for getting a deductively closed set of accepted beliefs from a confidence relation. Notice that $C E S$ and $C C S$ reduce to the $E S$ and $C S$ rules when $C=S$. These two properties will be the basis of our axiomatization of acceptance. Properties

\footnotetext{
${ }^{4}$ Our approach is in accordance with Bayes conditioning when $f$ is a probability, and using Dempster rule of conditioning (Shafer, 1976) when $f$ is a plausibility function or a possibility measure. It is preserved in qualitative possibility theory, which remains in the spirit of Cox's conditioning equation (Dubois and Prade, 1998), although conditional possibility derives from equality $f(A \cap C)=f(A \mid C) * f(C)$ with $*=$ minimum. However, this equation does not hold for the dual conditional functions of the form $f^{T}(A \mid B)=1-f(\neg A \mid B)$, when $f$ is a plausibility function or a possibility measure.
} 


\section{REPRESENTATIONS OF ACCEPTANCE}

described in the previous section, namely $M I, O$ and $Q T$ are also assumed but they concern the underlying confidence relation only, independently of the notion of accepted beliefs and their dynamics. However, notice that is not necessary to explicitly require $C E S$ for the confidence relation, since axiom $O$ clearly implies $C E S$. The latter is indeed axiom $O$ restricted to pairs of disjoint subsets $(A, B)$ and $\left(A^{\prime}, B^{\prime}\right)$ such that $A \subseteq A^{\prime}, B^{\prime} \subseteq B$ and $A \cup B=A^{\prime} \cup B^{\prime}$. Alternatively, from axiom $O, A>B \cup C$ implies $A \cup C>B$, which trivially implies $C E S$.

The last axiom we need is obviously related to the Success Postulate of belief revision theory : when an input information arrives in the form of a proposition $C$, the revised belief set $\boldsymbol{K}^{*} C$ contains $C$. In the framework of confidence relations, it makes sense to adopt this assumption, except when the context is contradictory (i.e. $C \neq \varnothing$ ). This is because the properties of confidence relations enforce $\boldsymbol{A}_{\mathrm{L}}(\varnothing)=\varnothing$. Contrary to classical logic, for which contradiction entails anything, we consider that, in the presence of contradictory information on the current situation, an agent cannot entertain beliefs. This is in the tradition of probability theory where conditioning on the empty set is not allowed. However, insofar as the agent considers that the information $C$ on the current situation is consistent and reliable, it is natural that $C$ becomes an accepted belief : in the context of confidence relations, the success postulate writes :

Success postulate (SUC) : $\forall C \neq \varnothing, C \in A_{L}(C)$.

This postulate implies that Definition 8 makes sense for confidence relations for which $C>_{L} \varnothing$ holds whenever $C \neq \varnothing$. At the formal level, it is possible to show that $C \in A_{\mathrm{L}}(C)$ only if $C>_{L} \varnothing$. Indeed SUC implies that $A_{\mathrm{L}}(C)$ is not empty when $C \neq \varnothing$. Hence, $A \cap C>_{L} \neg A \cap C$ for some $A$. Of course $A \neq \varnothing$, and by $O$, it follows that $C>_{L} \varnothing$. Hence, $A_{\mathrm{L}}(C) \neq \varnothing$ if and only if $C>_{L} \varnothing$ holds. A confidence relation such that $C>_{L} \varnothing$ holds whenever $C \neq \varnothing$ is called non-dogmatic.

Non-dogmatism : $\forall C \neq \varnothing, C>_{L} \varnothing$.

So, to obey the success postulate, it is necessary, and sufficient, to assume non-dogmatism for the confidence relation. For possibility relations, this condition means that no contingent proposition is impossible. Non-dogmatism is never verified for necessity relations since $C>_{N} \varnothing$ implies $\varnothing \geq_{\mathrm{N}} \neg C$. However if a confidence relation is non-dogmatic, its dual is such that $S>_{L}^{T} C$ holds whenever $C \neq S$. This is the condition requested by Gärdenfors for epistemic entrenchment. Non-dogmatism is natural in the framework of Gärdenfors' belief revision theory : it ensures that any non-tautological sentence may disappear upon a revision process. This restriction is not really a big issue since it is only a matter of choice between a confidence relation and its dual relation, which both represent the same information.

We can now define a formal ordinal framework that captures the concept of acceptance, that is, confidence relations that induce accepted beliefs:

Definition 9. An acceptance relation $\geq_{L}$ on $2^{S}$ is a non-dogmatic confidence relation that satisfies the CCS property.

The set of accepted beliefs according to an acceptance relation is then a consistent non-empty deductively closed set of propositions in any context. An acceptance relation is a priori not requested to be transitive nor complete. Transitive acceptance relations are called acceptance preorders. 


\subsection{Strict Acceptance Relations}

It is obvious that the properties that make the set of accepted beliefs (according to a confidence relation) a consistent non-empty deductively closed set only involve the strict part of the confidence relation, moreover restricted to disjoint subsets. The remaining part of the confidence relation has no influence on the set of accepted beliefs. The strict part of an acceptance relation is called a strict acceptance relation. It is irreflexive, transitive, non-dogmatic, and satisfies $O$ and $C C S$. When $z_{L}$ is complete and transitive, $>_{L}$ is necessarily irreflexive and transitive. More generally :

Definition 10: A strict acceptance relation (SAR) is an irreflexive, transitive and nondogmatic relation $>_{L}$ on $2^{S}$ that satisfies properties $O$ and $C C S$.

When only the strict part of the relation is available, there is a simple way of constructing a transitive acceptance relation from a relation between events verifying the properties of a SAR, by augmenting it using set-inclusion:

Proposition 1. If $>_{L}$ is irreflexive, transitive, non-dogmatic and satisfies $O$ and CCS then it is the strict part of an acceptance relation $\geq_{L}$ defined by $A \geq_{L} B$ if and only if $A>_{L} B$ or $B \subseteq A$. Relation $\geq_{L}$ is a transitive acceptance relation such that $\left(A \equiv_{L} B\right.$ if and only if $A=B$ ).

Proof : Suppose that $>_{L}$ is irreflexive and transitive and satisfies $\mathrm{O}$ and N. The relation $\geq_{L}$ satisfies MI by definition and its strict part is $>_{L}$. It obviously satisfies CCS. It is transitive since :

if $A>_{L} B$ and $B>_{L} C$, then $A>_{L} C$ and thus $A \geq_{L} C$;

if $A>_{L} B$ and $C \subseteq B$, then, by $O, A>_{L} C$ and thus $A \geq_{L} C$;

if $B \subseteq A$ and $B>_{L} C$, by $O, A>_{L} C$ and thus $A \geq_{L} C$;

if $B \subseteq A$ and $C \subseteq B, C \subseteq A$ and thus $A \geq_{L} C$.

Moreover, $A=B$ implies $A \equiv_{L} B$. Conversely, $A \equiv_{L} B$ means $\left(A>_{L} B\right.$ or $\left.B \subseteq A\right)$ and $\left(B>_{L} A\right.$ or $A \subseteq$ $B)$. Since $A \subseteq B$ implies that $\operatorname{not}(A>B)$, we have : $B \subseteq A$ and $A \subseteq B$.

Strict acceptance relations basically express negligibility. Indeed, as pointed out by Dubois and Prade (1995b), and Friedman and Halpern (1996), the following basic result yields yet another form of the CCS axiom:

Theorem 1: For any relation $>_{L}$ that satisfies $O, C C S$ is equivalent to the following negligibility property: $\forall A, B, C$ three disjoint events,

$$
\text { if } A \cup B>_{L} C \text { and } A \cup C>_{L} B \text {, then } A>_{L} B \cup C
$$

Proof : First, notice that CCS is equivalent to postulating that for disjoint subsets A, B, C, D: if $A \cup B>_{L} C \cup D$ and $A \cup C>_{L} B \cup D$ then $A>_{L} B \cup C \cup D$

Indeed, in CCS, let $B^{\prime}=A \cap C \cap \neg B, C^{\prime}=\neg A \cap C \cap B, A^{\prime}=A \cap B \cap C, D^{\prime}=C \cap \neg A \cap \neg B$. But $C \cap A=A^{\prime} \cup B^{\prime}, C \cap \neg A=C^{\prime} \cup D^{\prime}$, and $C \cap B=A^{\prime} \cup C^{\prime}$ and $C \cap \neg B=B^{\prime} \cup D^{\prime}$ : Then CCS is another way of writing (2). Now, CCS implies NEG, letting $D=\varnothing$ in (2).

Conversely, let us show that $\mathrm{O}$ and negligibility imply CCS: indeed, from $A \cup C>_{L} B \cup D$ and $\mathrm{O}$, we get $A \cup C \cup D>_{L} B$. Moreover, both $A \cup C \cup D>_{L} B$ and $A \cup B>_{L} C \cup D$ are of the form $A$ $\cup X>_{L} B$ and $A \cup B>_{L} X$ with $A, B, X$ disjoint. So, by negligibility, $A>_{L} B \cup C \cup D$, which is (2). 


\section{REPRESENTATIONS OF ACCEPTANCE}

The negligibility property can be explained as follows : for three disjoint events $A, B, C, A>{ }_{L} C$ and $A>_{L} B$ means that each of $B$ and $C$ is less plausible than $A$. However, whatever their respective plausibility levels, their disjunction is always less plausible than A since, from $\mathrm{O}$, it follows $A \cup B>_{L} C$ as well as $A \cup C>_{L} B$, and, by NEG, $A>_{L} B \cup C$. So $A>_{L} C$ means that the confidence in $C$ is negligible in front of the confidence in $A$. If $>_{L}$ derives from a probability function, the negligibility property certainly does not hold in general. However this property is valid when $>_{L}$ derives from a possibility relation. This idea of negligibility between the likelihood levels of events was put forward by Lehmann (1996). From a probabilistic perspective property NEG is debatable if not very strange. However, it is absolutely needed if accepted beliefs are to form a deductively closed set. It also suggests that for $A$ to be accepted and reasoned with, there must be a big likelihood gap between A and its negation. From this point of view, property NEG is on the contrary acknowledging the intuitive idea that, for a proposition to be accepted, it must be firmly entrenched.

So-called qualitative relations in the sense of Halpern (1997) satisfy $N E G$. Recall that a relation $>$ on $2^{S}$ is called "qualitative" when

$$
\forall A, B, C \text { : If } A \cup B>C \text { and } A \cup C>B \text {, then } A>B \cup C \quad \text { (Qual) }
$$

Qual is much stronger than NEG since it applies to any sets $A, B, C$ (not necessarily disjoint). Any irreflexive qualitative orderly relation is necessarily transitive (Halpern, 1997) and is thus a SAR. We will show in the next section that such SAR's play a particular role. In the context of complete preorderings, requiring Qual precisely yields possibility relations, since

Proposition 2. For complete and transitive confidence relations, property Qual is equivalent to unrestricted union stability (the characteristic axiom of possibility relations).

Proof : Possibility relations are complete and transitive confidence relations. Using their representation by possibility measures, possibility relations satisfy Qual because the property $\max (a, b)>c$ and $\max (a, c)>b$ implies $a>\max (b, c)$ trivially holds. Conversely if Qual holds, assume $A \cup B>A \cup C$. Then by $O, A \cup B>C$ and $A \cup B>A$. Applying Qual to the latter implies $B$ $>A$. By $O$, it implies $B \cup C>A$. Applying Qual again to the latter and $A \cup B>C$ yields $B>C$ as a consequence of $A \cup B>A \cup C$, and by contraposition, it gives the characteristic axiom of possibility relations.

Several useful examples of acceptance relations can be given. They are used in the sequel.

A strict acceptance relation can be obtained considering a family $\boldsymbol{F}$ of possibility relations, and letting $A>_{\mathbf{F}} B$ if and only if $A>_{\Pi} B$ for all $\geq_{\Pi}$ in $\boldsymbol{F}$. Like all the SAR's, $>_{\mathbf{F}}$ is transitive but, contrary to the strict part of a possibility relation, this relation is not negatively transitive. The negative transitivity of a relation (If $A>_{L} B$, then $C>_{L} B$ or $A>_{L} C$ ) is equivalent, by contraposition to the transitivity of the negation of this relation.

Different acceptance relations can share the same SAR. For instance, the previous relation $>_{\mathbf{F}}$ can be the strict part of different acceptance relations, e.g. the acceptance relation defined by letting $A \geq_{F} B$ if and only if $\operatorname{not}\left(A>_{\boldsymbol{F}} B\right)$, which is complete but not transitive; and also the acceptance relation defined by letting $A \geq_{\boldsymbol{F}} B$ if and only if $A>_{\Pi} B$ for all $\geq_{\Pi}$ in $\boldsymbol{F}$ or $\mathrm{A} \equiv_{\Pi} \mathrm{B}$ for all $\geq_{\Pi}$ in $\boldsymbol{F}$, which is not complete but is transitive. 


\section{DUBOIS, FARGIER, \& PRADE}

Some acceptance relations are preadditive. For instance, let $\Pi$ be a possibility function and consider the strict ordering $>_{\Pi L}$ and the relation $\geq_{\Pi L}$ called possibilistic likelihood, and introduced by Dubois et al. (1997) :

$$
\begin{gathered}
A>_{\Pi L} B \text { if and only if } A \cap \neg B>_{\Pi} \neg A \cap B \text { if and only if } A \cup \neg B>_{N} B \cup \neg A ; \\
\qquad A \geq_{\Pi L} B \text { if and only if } \operatorname{not}\left(B>_{\Pi L} A\right) .
\end{gathered}
$$

$>_{\Pi L}$ is a self-dual SAR and $\geq_{\Pi L}$ is an acceptance relation. $>_{\Pi L}$ refines $>_{\Pi}\left(A>_{\Pi} B\right.$ implies $A>_{\Pi L}$ $B$ ) comparing only the non-common part of events : if $A$ and $B$ are disjoint, $A>_{\Pi L} B$ is identical to $A>_{\Pi} B$, and then $A_{\Pi(}(C)=A_{\Pi L}(C)$. But $\geq_{\Pi L}$ is generally not transitive : $\Pi(A \cap \neg B)=\Pi(\neg A \cap B)$ and $\Pi(B \cap \neg C)=\Pi(\neg B \cap C)$ do not imply $\Pi(A \cap \neg C)=\Pi(\neg A \cap C)$. The same construction works with a family of possibility relations. Notice that the SAR $>_{\Pi L}$ does not obey axiom Qual. Consider for instance $S=\left\{s_{1}, s_{2}\right\}$ and a possibility measure such that $\left\{s_{1}\right\}>_{\Pi}\left\{s_{2}\right\}>_{\Pi} \varnothing$. It holds that $\left\{s_{1}, s_{2}\right\}>_{\Pi L}\left\{s_{1}\right\}>_{\Pi L}\left\{s_{2}\right\}>_{\Pi L} \varnothing$. So $:\left\{s_{1}\right\} \cup\left\{s_{1}\right\}>_{\Pi L}\left\{s_{2}\right\}$ and $\left\{s_{1}\right\} \cup\left\{s_{2}\right\}>_{\Pi L}\left\{s_{1}\right\}$ but $\left\{s_{1}\right\}>_{\Pi L}$ $\left\{s_{1}\right\} \cup\left\{s_{2}\right\}$ does not hold. However, the relative position of $\left\{s_{1}, s_{2}\right\}$ w.r.t. $\left\{s_{1}\right\}$ (higher or at the same rank) is immaterial for characterizing accepted beliefs.

\subsection{Acceptance Relations and Comparative Probabilities}

Comparative probabilities are preadditive and complete confidence preorders. They are more general than confidence relations induced by probabilities. Probability measures generally do not induce acceptance relations. It is thus interesting to characterize comparative probabilities modeling acceptance. For a preaddidive and complete confidence preorder $\geq_{L}$, it holds, for any $A$, $B, C$ such that $A \cap(B \cup C)=\varnothing$, that $A \cup B \equiv_{L} A \cup C$ iff $B \equiv_{L} C$, and $A \cup B>_{L} A \cup C$ iff $B>_{L} C$.

In the context of transitive acceptance relations, it turns out that there is a strong incompatibility between the existence of equally plausible disjoint events and preadditivity. Indeed, such acceptance relations have the following property :

Lemma 1. Let $A, B$ and $C$ be three disjoint events. If $\geq_{L}$ is a complete and transitive acceptance relation, then $A \equiv_{L} C>_{L} B$ implies $A \equiv_{L} A \cup B \equiv_{L} C \equiv_{L} C \cup B>_{L} B$.

Proof : $C>_{L} B$ implies, by $O, C \cup A>_{L} B$. Moreover, $C \geq_{L} A$ also implies $A \cup B \geq_{L} C$. And $A \cup B$ $>_{L} C$ is impossible (otherwise, by $N E G, A>_{L} C \cup B$ and thus $A>_{L} C$ by $O$ ). So, $A \cup B \equiv_{L} C$, i.e. by transitivity $A \cup B \equiv_{L} A$.

Lemma 1 makes it clear that when $A>_{L} B$, the plausibility of $B$ is negligible when compared to the one of $A$ since $A \equiv_{L} A \cup B$. However, under transitivity, preadditivity will severely constrain the possibility of expressing negligibility between events. Indeed, $B>_{L} \varnothing$ holds for acceptance relations if $B \neq \varnothing$. So $A \cup B>_{L} A$ is enforced by preadditivity for any two disjoints nonempty sets $\mathrm{A}$, and B. It contradicts the property $A \equiv_{L} A \cup B$ enforced by the presence of another disjoint set $C \equiv_{L} A$. More formally :

Lemma 2. If $\geq_{L}$ is a preadditive, complete and transitive acceptance relation, then for any three disjoint events $A, B$ and $C: C \equiv_{L} A>_{L} B$ implies $B=\varnothing$. 


\section{REPRESENTATIONS OF ACCEPTANCE}

Proof : Clearly, $A \neq \varnothing$. $C \equiv_{L} A>B$ implies $A B \equiv_{L} A \cup B$ by Lemma 1. By preadditivity, $B \equiv_{L}$ $\varnothing$. But non-dogmatism enforces $B=\varnothing$.

Hence as soon as equivalence is allowed between disjoint events, acceptance relations that are preadditive complete preorderings have to be very particular : no state can be less plausible than two equivalent states, except if it is impossible. In summary, assuming transitivity, preadditivity is little compatible with negligibility. The conflict between these two properties makes deductive closure hard to reconcile with the notion of partial belief graded on a numerical scale. Clearly the following result follows for comparative probabilities :

Theorem 2. If an acceptance relation $\geq_{L}$ is a comparative probability on $S$ and $S$ has more than two elements, then, there is a permutation of the elements in $S$, such that $s_{1}>_{L} s_{2}>_{L} \ldots$ $>_{L} S_{n-1} \geq_{L} s_{n}$.

Proof: From the above results, if $s_{i}>_{L} s_{k}$ and $s_{j}>_{L} s_{k}$ we have that either $s_{i}>_{L} s_{j}$ or $s_{j}>_{L} s_{i}$. Hence the equally plausible states are the least plausible ones. Suppose now that there are more that one least plausible states. Assume $\left\{s_{i}, s_{j}\right\} \equiv_{L}\left\{s_{i}\right\}$; then $\varnothing \equiv_{L}\left\{s_{i}\right\}$, which is impossible since the relation is not dogmatic. Hence $\left\{s_{i}, s_{j}\right\}>_{L}\left\{s_{i}\right\}$. Hence, in the set of least plausible states, events are strictly ordered in agreement with set-inclusion. Suppose there are more than two equally plausible states $s_{i}, s_{j}, s_{k}$. Then preadditivity implies $\left\{s_{i}, s_{j}\right\}>_{L}\left\{s_{k}\right\}$ and $\left\{s_{i}, s_{k}\right\}>_{L}\left\{s_{j}\right\}$, which due to (NEG) implies $\left\{s_{i}\right\}>_{L}\left\{s_{k}, s_{j}\right\}$, and this is impossible since by assumption states $s_{i}, s_{j}, s_{k}$. are equally plausible. Hence there cannot be more than two equally plausible states, and these are the least plausible ones, if any.

Note that the possibilistic likelihood relation $\geq_{\Pi L}$ of Section 2.5 is a preadditive acceptance relation, but it is not transitive, so it allows for equally plausible states and escapes Theorem 2.

The problem of finding probability measures that are acceptance functions can be easily solved on this basis. Necessarily the states are totally ordered (but for the two least probable ones). Moreover such comparative probabilities coincide with possibility relations on disjoint events A and B: $P(A)>P(B)$ if and only if $\Pi(A)>\Pi(B)$, that is $\max _{i \in A} p_{i}>\max _{i \in B} p_{i}$. A necessary and sufficient condition for this probability-possibility compatibility is that $\forall A \exists s \in A$ such that $P(\{s\})>P(A \backslash\{s\})$. This leads to very special probability measures such that the probability of a state is much bigger than the probability of the next probable state (Snow, 1999; Benferhat et al., 1999a) - they are called "big-stepped" probabilities. They are lexicographic probabilities. It is clear that any comparative probability that is an acceptance function can be represented by any big-stepped probability $\mathrm{P}$ such that :

$$
\begin{gathered}
p_{i}=P\left(\left\{s_{i j}\right\}\right): p_{1}>\ldots>p_{n-1} \geq p_{n}>0 .{ }^{5} \\
\forall i<n-1, p_{i}>\Sigma_{j=i+1, \ldots n} p_{i} .
\end{gathered}
$$

Example : $S$ has four elements and consider $s_{1} / 0.6, s_{2} / 0.3, s_{3} / 0.06 /, s_{4} / 0.03, s_{5} / 0.01$.

\footnotetext{
${ }^{5}$ The weak inequality $p_{n-1} \geq p_{n}$ reflects the corresponding result in Theorem 2 . In fact, on a 2-element set, equal probabilities are compatible with acceptance and the corresponding belief set only contain a tautology (S).
} 


\section{Representing Strict Acceptance Relations by Possibility Relations}

In the examples of Section 2.5, it was shown that a family of possibility relations generates an acceptance relation. In this section, we prove the converse, namely that, for any acceptance relation, there exists a family of possibility relations, such that the set of accepted beliefs derived from the acceptance relation is the intersection of the sets of accepted beliefs derived from the possibility relations in the family. This result clearly establishes that the notion of acceptance is basically compatible with possibility theory and virtually nothing else, if we except very special probability measures met in the previous section, whose behavior makes them very similar to possibility measures.

\subsection{The Cautious Substitute to a Strict Acceptance Relation}

First, let $\geq_{L}$ be an acceptance relation on the subsets of $S$, and let $A_{\mathrm{L}}(C)$ be the sets of accepted beliefs in context $C$. Clearly, $A_{\mathrm{L}}(C)$ does not depend on the entire acceptance relation, nor on its entire strict part, but only on the restriction of its strict part to disjoint events. This restriction is called the disjoint graph.

Definition 11: The disjoint graph of an irreflexive relation $>$ on events, denoted $G(>)$ is defined by $G(>)=\{(A, B), A>B$ and $A \cap B=\varnothing\}$.

It directly follows from this definition that two SAR's $>_{L}$ and $>_{M}$ that share the same disjoint graph also share the same set of accepted beliefs : $G\left(>_{L}\right)=G\left(>_{M}\right)$ implies $\forall C \subseteq S, A_{\mathrm{L}}(C)=$ $\boldsymbol{A}_{\mathrm{M}}(C)$. Two SARs having the same disjoint graph will be called acceptance-equivalent. Of course, this property defines an equivalence relation among SARs. Our purpose is to show that, for any SAR $>_{L}$, there exists another SAR $>$ such that $G(>)=G\left(>_{L}\right)$ (hence yielding the same sets of accepted beliefs in all contexts) that can be represented by a family of possibility relations and this is precisely, in the acceptance-equivalent class of $>_{L}$, the SAR that satisfies Halpern's Axiom of Qualitativeness ( $A>B$ and $A>C$ imply $A>B \cup C$, without any restriction).

The idea is to build a SAR $>$, acceptance-equivalent to $>_{L}$, that satisfies the following property of called semi-cancellativeness:

$$
A>B \text { if and only if } A \cap \neg B>B
$$

We will see at the end of this section that, provided that $O$ holds, this property is equivalent to Qual, with the advantage of being more tractable. Indeed, it allows an easy and constructive definition of $>$ from $>_{L}$.

Definition 12. Let $>_{L}$ be a SAR. The relation $>$ defined by: $\forall A, B \subseteq S, A>B$ if and only if $A \cap \neg B>_{L} B$ is called the (cautious) substitute of $>_{L}$.

$>$ and $>_{L}$ are different relations in general, since many SAR do not satisfy $S C$, while the relations built from Definition 12 do (by construction).

Example : The SAR $\left\{s_{1}, s_{2}\right\}>_{L}\left\{s_{1}\right\}>_{L}\left\{s_{2}\right\}>_{L} \varnothing$ does not satisfy SC (since $\left\{s_{1}, s_{2}\right\}>_{L}\left\{s_{1}\right\}$ holds while $\left\{s_{2}\right\}>_{L}\left\{s_{1}\right\}$ does not hold) and its substitute is : $\left\{s_{1}, s_{2}\right\}>\left\{s_{2}\right\} ;\left\{s_{1}\right\}>\left\{s_{2}\right\} ;\left\{s_{1}\right\}>\varnothing ;\left\{s_{2}\right\}>$ 


\section{REPRESENTATIONS OF ACCEPTANCE}

$\varnothing .>_{L}$ is actually a refinement of $>^{6}$, (indeed, by construction $A>B$ implies $A \cap \neg B>_{L} B$, which due to $O$, also implies $A>_{L} B$ ).

The new relation $>$ is denoted $\operatorname{cs}\left(>_{L}\right)$. It has very remarkable properties. First, it is a SAR, obviously acceptance-equivalent to $>_{L}$ (indeed, $G\left(\operatorname{cs}\left(>_{L}\right)\right)=G\left(>_{L}\right)$ since $\operatorname{cs}\left(>_{L}\right)=>_{L}$ on disjoint subsets) :

Proposition 3. Let $>_{L}$ be a SAR. The relation $>=c s\left(>_{L}\right)$ defined by $A>B$ if and only if $A \cap \neg B>_{L} B$ is a SAR i.e. non-dogmatic, irreflexive, orderly, transitive and it satisfies NEG.

Proof : since $G(>)=G(>L)$, > is non dogmatic, and satisfies NEG.

Irreflexivity : suppose $A>A$. Due to (SC), $A>A$ is equivalent to $\varnothing>_{L} A$, which implies $\varnothing>_{L} \varnothing$ via $O$. This is impossible since $>_{L}$ is irreflexive.

Orderly : Suppose $A>B \cup C$, for B and C disjoint. Due to (SC), $\mathrm{A} \cap \neg \mathrm{B} \cap \neg \mathrm{C}>_{L} \mathrm{~B} \cup \mathrm{C}$. Since $>_{L}$ is orderly, it immediately follows that $A \cap \neg B>>_{L} B$, which is $A>B$. Similarly, suppose $A>B$, that is $\mathrm{A} \cap \neg \mathrm{B}>_{L} \mathrm{~B}$. Since $>_{L}$ is orderly, it immediately follows that $(\mathrm{A} \neg \neg \mathrm{B}) \cup(\mathrm{C} \cap \neg \mathrm{B})>_{L} \mathrm{~B}$, which is $\mathrm{A} \cup \mathrm{C}>\mathrm{B}$.

Transitivity : Suppose $A>B$ and $B>C$. It reads $A \cap \neg B>_{L} B$ and $B \cap \neg C>_{L} C$. It implies $(\mathrm{A} \cup \mathrm{C}) \cap \neg \mathrm{B}>_{L} \mathrm{~B}$ and $(\mathrm{A} \cup \mathrm{B}) \cap \neg \mathrm{C}>_{L} \mathrm{C}$, via $O$. Note that $(A \cup C) \cap \neg B=(A \cap \neg B \cap \neg C) \cup(\neg B \cap C)$ and $(A \cup B) \cap \neg C=(A \cap \neg B \cap \neg C) \cup(B \cap \neg C), B=(\neg B \cap C) \cup(B \cap C)$ and $C=(B \cap \neg C) \cup(B \cap C)$. Applying NEG yields $A \cap \neg B \cap \neg C>_{L} B \cup C$. Then applying $\mathrm{O}$ again $: A \cap \neg C>_{L} C$, which is $A>C$.

Notice that different SARs can share the same substitute; actually any pair of acceptanceequivalent SAR share the same substitute, since Definition 12 builds the substitute from the disjoint graph, by the simple application of axiom $\mathrm{O}$ :

Proposition 4. Let $>_{L}$ and $>_{M}$ be two SAR: $\operatorname{cs}\left(>_{M}\right)=\operatorname{cs}\left(>_{L}\right)$ if and only if $G\left(>_{M}\right)=G\left(>_{L}\right)$.

Obviously, $\operatorname{cs}\left(\operatorname{cs}\left(>_{L}\right)\right)=\operatorname{cs}\left(>_{L}\right)$ because if a SAR $>_{L}$ satisfies SC, then $c s\left(>_{L}\right)=>_{L}$. It follows that $\operatorname{cs}\left(>_{L}\right)$ is the intersection of all SAR's $>_{M}$ such that $\operatorname{cs}\left(>_{M}\right)=\operatorname{cs}\left(>_{L}\right)$. So the substitute $\operatorname{cs}\left(>_{L}\right)$ is the most cautious, the least informative relation that determines the same accepted beliefs as $>_{L}$ in all contexts. That is why $\operatorname{cs}\left(>_{L}\right)$ can be named the cautious substitute of $>_{L}$.

Semi-cancellative SARs are very close to possibilistic relations, more precisely they generalize them to the case of partial orders. Indeed, most of the important properties of possibility relations are easily established for a semi-cancellative SAR, and above all their characteristic axiom (in its contraposed form, since we deal here with strict orders):

Proposition 5: Let $>$ be a semi-cancellative SAR.. Then $A \cup B>A \cup C$ implies $B>C, \forall A$, $B, C \subseteq S$.

Proof : by SC, $A \cup B>A \cup C$ is equivalent to $(A \cup B) \neg A \cap \neg C>A \cup C$. Since $(A \cup B) \cap \neg A \cap \neg C=$ $B \cap \neg A \cap \neg C, B>C$ follows by $\mathrm{O}$.

Proposition 6. Let $>$ be a semi-cancellative SAR. Then $A>B$ and $A>C$ imply $A>B \cup C$, without any restriction.

\footnotetext{
${ }^{6}$ By definition, a relation $>$ ' is a refinement of a relation $>$ iff $\forall \mathrm{A}, \mathrm{B}, \mathrm{A}>\mathrm{B} \Rightarrow \mathrm{A}>{ }^{\prime} \mathrm{B}$
} 
Proof : $A>B$ and $A>C$ is equivalent to $A \cap \neg B>B$ and $A \cap \neg C>C$. By $\mathrm{O}$, it implies $(A \cup C) \neg B>$ $B$ and $(A \cup B) \neg C>C$. This is just like in the proof of the transitivity of $>$. So, $A \cap \neg B \cap \neg C>B \cup C$. Then applying $\mathrm{O}$ again : $A>B \cup C$.

An important consequence of this property is that $A \cup B>B$ and $A \cup B>A$ cannot simultaneously hold (otherwise, by Proposition 6, $A \cup B>A \cup B$ ). A useful corollary follows :

Proposition 7. Let $\geq_{L}$ an acceptance relation the strict part of which is semi-cancellative. Then, $\forall A \neq \varnothing, \exists s \in A$, such that $\left(A>_{L}\{s\}\right)$ does not hold.

Lastly, let us check that qualitative SARs prove to be the same as semi-cancellative SARs :

Theorem 3. For any orderly relation > on events, the two properties Qual and SC are equivalent.

Proof : SC implies Qual: Obvious using proposition 6 and axiom O. Qual implies SC : $A>B$ implies $A \cup B>A \cap B$ by O. It writes $(A \cap \neg B) \cup B>A \cap B . A>B$ also writes $(A \cap \neg B) \cup(A \cap B)$ $>B$. Applying Qual leads to $A \cap \neg B>B$.

In summary, the semi-cancellative SAR obtained by Definition 12 is an acceptance-equivalent and qualitative substitute to the original SAR and has a very possibilistic flavor. We show in the next section that it can be represented by a family of possibility relations.

\subsection{Beliefs Induced by Strict Acceptance Relations can be Represented by Possibility Relations}

Given a family $\boldsymbol{F}$ of possibility relations, the strict confidence relation $>_{\mathbf{F}}$ defined by $A>_{\mathbf{F}} B$ if and only if $A>_{\Pi} B$ for all $\geq_{\Pi}$ in $\boldsymbol{F}$ is clearly a semi-cancellative SAR. The rest of this section will show that any semi-cancellative SAR $>$ can be represented by a family of possibility relations. More precisely we show that for any semi-cancellative SAR $>$, there is a non-empty family $\boldsymbol{F}$ of possibility relations such that $A>B$ if and only if $A>_{\Pi} B$ for all $\geq_{\Pi}$ in $\boldsymbol{F}$.

So, let $>$ be a semi-cancellative SAR, the cautious substitute of some SAR $>_{L}$. Since it is irreflexive and transitive, the strict order $>$ is a directed acyclic graph with node set $2^{S}$. This graph is not necessarily connected, but there are no isolated nodes in the graph since $A>\varnothing$ for $A \neq \varnothing$. Hence it is possible to assign a $\operatorname{rank} R^{*}(A)$ to each subset $A$ by letting

$$
\begin{gathered}
R^{*}(A)=1 \text { if }\{B, B>A\}=\varnothing\left(\text { e.g. } R^{*}(S)=1\right) \text { and } \\
R^{*}(B)=\max _{A: A>B} R^{*}(A)+1 .
\end{gathered}
$$

Notice that this ranking is unique. This is a well-known algorithm for acyclic directed graphs (Bellman et al., 1970). This construction is also used by Pearl (1990). It yields a well-ordered partition (wop) of $2^{S}$, say $\left\{\Psi_{1}, \Psi_{2}, \ldots \Psi_{k}, \Psi_{k+1}\right\}$ such that :

$$
\Psi_{k+1}=\{\varnothing\}
$$




\section{REPRESENTATIONS OF ACCEPTANCE}

$$
A \in \Psi_{i} \text { if and only if } R^{*}(A)=i, i=1, \ldots k
$$

By construction, $A>B$ if and only if $A \in \Psi_{i}, B \in \Psi_{j}, i<j$. And $R^{*}(A) \leq R^{*}(\{s\}), \forall s \in A$. However, by Proposition 7, we know that $A>\{s\}$ cannot hold for all $s \in A$, since $>$ is a semicancellative SAR. Hence :

Lemma 3. $R^{*}(A)=R^{*}\left(\left\{s_{\}}\right\}\right.$for some $s \in A$

Proof : let us first show that $R^{*}(A) \leq R^{*}(\{s\}) \forall s \in A$; indeed, by $\mathrm{O}$, it holds that $B>A$ and $C \subseteq A$ implies $B>C$, so the number of predecessors of any subset of $A$ is at least equal to the number of predecessors of $A$. Hence, its rank is at least equal to the rank of $A$. Let us prove now by recursion that for any rank k: $\forall A \subseteq S$, such that $R^{*}(A)=k, \exists s \in A, R^{*}(\{s\})=R^{*}(A)$.

For $k=1: R^{*}(A)=1$ implies $R^{*}(\{s\})=1$ for some $s \in A$. For suppose not. Then $R^{*}(\{s\})>R^{*}(A)=1$ for all $s \in A$. Since $R^{*}(A)=1$, there is no $B>A$. But there is some $B_{S}>\{s\}$ for all $\mathrm{s} \in A$. Hence $\cup_{S \in A} B_{S}>A$ using Qual. So $R^{*}(A)>1$. Contradiction.

Suppose that the property holds up to rank $k-1: \forall i=1, \ldots, k-1, R^{*}(A)=i$ implies $R^{*}\left(\left\{s_{\}}\right\}\right)=i$ for some $s \in A$. Then, $R^{*}(A)=k$ implies $R^{*}(\{s\})=k$ for some $s \in A$. For suppose not. Then, for some set $A, R^{*}\left(\left\{s_{\}}\right)>R^{*}(A)=k\right.$ for all $s \in A$. So, there is some $B_{S}>\{s\}$, with $R^{*}\left(B_{S}\right)=k$, for all $\mathrm{s} \in A$. Hence $B=\cup_{S} \in A B_{S}>A$ using Qual. So $R^{*}(B)<k$. Using the recursivity assumption, $R^{*}\left(\left\{s^{\prime}\right\}\right)=R^{*}(B)<k$ for some $s^{\prime} \in B$. Such a state $s^{\prime}$ belongs to some set $B_{S}$. And $R^{*}\left(B_{S}\right) \leq R^{*}\left(\left\{s^{\prime}\right\}\right)$ $<k$. Contradiction, since $R^{*}\left(B_{S}\right)=k$, for all $\mathrm{s} \in A$.

From this Lemma, it follows that $\forall A \subseteq S, R^{*}(A)=\min _{S} \in R^{*}(\{s\})$. It is then easy to see that : $\forall A, B, C, R^{*}(A \cup B)>R^{*}(A \cup C)$ implies $R^{*}(B)>R^{*}(C)$. Hence, we have proved that :

Proposition 8. The relation $\geq_{\Pi *}$ on subsets induced by $R^{*}(A): A \geq_{\Pi *} B$ if and only if $R^{*}(A)$ $\leq R^{*}(B)$ is a possibility relation.

The corresponding plausibility preorder $\left(S, \geq_{\pi^{*}}\right)$ on states is trivially given by $: s \geq_{\pi^{*}} s^{\prime}$ iff $R^{*}(\{s\}) \leq R^{*}\left(\left\{s^{\prime}\right\}\right)$. Now let us define from the ranking $R^{*}$ (or equivalently from $\geq_{\pi^{*}}$ ) a well ordered partition of $S$, say $\left\{E_{1}, E_{2}, \ldots E_{m}\right\}$, such that $E_{i}=\left\{s, R^{*}(\{s\})=i\right\}, i=1, \ldots \max _{S} R^{*}(\{s\}) . E_{1}$ contains the most plausible states by default, $E_{2}$ the second most plausible states, etc.

It is obvious that the strict possibility relation $>_{\Pi *}$ corresponding to the ranking $\mathrm{R}$ refines $>$ since, by construction, $A>B$ implies $R^{*}(A)<R^{*}(B)$, which in turn implies $A>_{\Pi *} B$. In fact, generally, relation $>_{\Pi *}$ contains more pairs than $>$. Suppose for instance that $S=\left\{s_{1}, s_{2}, s_{3}\right\}$, and consider the semi-cancellative SAR induced by the only constraint $\left\{s_{1}, s_{2}\right\}>\left\{s_{3}\right\}$. Then $\Psi_{3}=\{\varnothing\}, \Psi_{2}=\left\{s_{3}\right\}$, $\Psi_{1}$ contains all other events. Then $E_{1}=\left\{s_{1}, s_{2}\right\}$ and $E_{2}=\left\{s_{3}\right\}$. Hence, $R^{*}\left(\left\{s_{2}\right\}\right)<R^{*}\left(\left\{s_{3}\right\}\right)$, thus $\left\{s_{2}\right\}>_{\Pi *}\left\{s_{3}\right\}$ while $\left\{s_{2}\right\}>\left\{s_{3}\right\}$ does not hold.

At this point we can construct a family of possibility relations from a semi-cancellative SAR: let $\boldsymbol{F}(>)$ be the set of all possibility relations $\geq_{\Pi}$ whose strict part refines the semi-cancellative SAR $>$. More formally : $\boldsymbol{F}(>)=\left\{\geq_{\Pi}\right.$ such that $\geq_{\Pi}$ is a possibility relation and $A>B$ implies $A>_{\Pi}$ $B$. What has been proved above is that $\boldsymbol{F}(>) \neq \varnothing$ since $\boldsymbol{F}(>)$ contains $\geq_{\Pi * *}$. Note that $A>_{\Pi} B$ holds provided that $\exists s \in A \cap \neg B, \forall s^{\prime} \in B, s>_{\pi} s^{\prime}$. So $\boldsymbol{F}(>)$ can be identified with the set of all the complete preorderings $\geq_{\pi}$ on states such that, for any pair of disjoint subsets $(A, B)$, if $A>B$ then $\exists s \in A, \forall s^{\prime} \in B, s>_{\pi} s^{\prime}$. 


\section{DUBOIS, FARGIER, \& PRADE}

Let us compare the possibility relations in $\boldsymbol{F}(>)$, in terms of their relative informativeness. A preorder $\left(S, \geq_{\pi}\right)$ is said to be more specific than $\left(S, \geq_{\mu}\right)$ in the wide sense if and only if, in terms of their associated wops $\left\{E_{1}, E_{2} \ldots E_{m}\right\}$ and $\left\{F_{1}, F_{2} \ldots F_{k}\right\}$ respectively, it holds that (Benferhat et al. 1999b):

$$
\forall i=1, \min (m, k), E_{1} \cup E_{2} \cup \ldots \cup E_{i} \subseteq F_{1} \cup F_{2} \cup \ldots \cup F_{i}
$$

The idea is that the specificity of a plausibility preorder on $\mathrm{S}$ is decreased by moving states up towards more plausible elements of a wop. Maximally specific wops are the same as maximally refined wops and correspond to linear orderings of states. The least specific wop is also the least refined one, made of $\mathrm{S}$ itself. However the specificity ordering differs from the refinements of wops. In terms of ranking functions, $\geq_{\pi}$ is less specific than $\geq_{\mu}$ in the wide sense if and only if $\forall s$, $R_{\pi}(s) \leq R_{\mu}(s)$, where $R_{\pi}(s)$ (resp. $R_{\mu}(s)$ ) is the rank of state $s$ given the complete preordering $\geq_{\pi}$ (resp. $\geq_{\mu}$ ). Decreasing specificity makes a ranking more compact (Pearl, 1990).

The following proposition shows that $\geq_{\Pi^{*}}$, obtained by the ranking $R^{*}$ of subsets induced by the SAR $>$, is also the least specific possibility relation in $\boldsymbol{F}(>)$. We do it by showing that $R^{*}$ is the most compact ranking of states induced by the semi-cancellative SAR.

Proposition 9. $\geq_{\Pi} \in \boldsymbol{F}(>)$ implies that $\geq_{\Pi}$ is more specific than $\geq_{\Pi *}$ in the wide sense.

Proof : Let $\mathrm{R}$ be the ranking function of a possibility relation $\geq_{\Pi}$. This possibility relation extends the SAR $>$ if and only if $A>B$ implies $R(A)<R(B) \forall A, \forall B$. Hence $R(B) \geq \max _{A}: A>B R(A)+1$. In particular, for $>_{\Pi^{*},}$ note that $R^{*}(B)=\max _{A}: A>B R^{*}(A)+1$. By construction, sets without predecessors with respect to ordering $>_{\Pi}$. should be chosen among the ones without predecessors with respect to $>$. Hence $R(A)=1$ implies $R^{*}(A)=1$. Now assume $R(A) \geq R^{*}(A)$ whenever $R^{*}(A)$ $<k$. Suppose B such that $R^{*}(B)=k$. So, if $A>B, R^{*}(A)<k$.

Then $R(B) \geq \max _{A}: A>B R(A)+1 \geq \max _{A}: A>B R^{*}(A)+1=k$.

However the converse of Proposition 9 does not hold. Namely if $\geq_{\Pi}$ is more specific than $\geq_{\Pi *}$ in the wide sense then $\geq_{\Pi} \in \boldsymbol{F}(>)$ is not warranted.

Example : Consider $S=\left\{s_{1}, s_{2}, s_{3}\right\}$, and the semi-cancellative SAR $>$ induced by the only constraints $\left\{s_{1}, s_{2}\right\}>\left\{s_{3}\right\}$ and $\left\{s_{2}\right\}>\left\{s_{3}\right\} . \geq_{\pi^{*}}$ corresponds to the two levels partition where $E_{1}=$ $\left\{s_{1}, s_{2}\right\}$ and $E_{2}=\left\{s_{3}\right\}$. The possibility relation induced by the plausibility order $s_{1}>_{\pi} s_{3}>_{\pi} s_{2}$ is more specific than $\geq_{\pi^{*}}$ but is not a refinement of $>$ (and thus $>_{\Pi}$ does not belong to $\boldsymbol{F}(>)$ ).

Now, among possibility relations in $\boldsymbol{F}(>)$, some induce a linear order on S.

Definition 13. A linear possibility relation is a possibility relation $>_{\Pi}$ such that for any disjoint $A, B$ either $A>_{\Pi} B$ or $B>_{\Pi} A$ holds.

It is obvious that the well-ordered partition of states associated to a linear possibility relation contains singletons only and its associated plausibility ordering of states is a total ranking of S. Consider a total ranking of $S$ obtained by concatenating arbitrary total rankings of elements in the sets $E_{i}$ 's taken the wop induced by a semi-cancellative SAR $>$. It respects the preorder induced by the wop and is a refinement of the wop $\left\{E_{1}, E_{2}, \ldots E_{m}\right\}$. And it yields a linear possibility relation that refines $>_{\Pi *}$, hence it refines $>$ and lies in $\boldsymbol{F}(>)$. 


\section{REPRESENTATIONS OF ACCEPTANCE}

Let $\boldsymbol{F}_{\boldsymbol{l}}(>)=\left\{\geq_{\Pi}\right.$ linear and $A>B$ implies $\left.A>_{\Pi} B\right\}$ be the set of linear possibility relations in $\boldsymbol{F}(>)$ for a semi-cancellative SAR. We have seen that this set is not empty, since it contains at least linear refinements of $>_{\pi^{*}}$. Notice that it generally contains more than the linear refinements of the wop $\left\{E_{1}, E_{2}, \ldots E_{m}\right\}$ of $\geq_{\pi^{*}}$.

Example : Consider again $S=\left\{s_{1}, s_{2}, s_{3}\right\}$, and the semi-cancellative $S A R>$ induced by the only constraint $\left\{s_{1}, s_{2}\right\}>\left\{s_{3}\right\}$. Then $E_{1}=\left\{s_{1}, s_{2}\right\}$ and $E_{2}=\left\{s_{3}\right\}$. The linear refinements of the wop are $s_{1}>\pi s_{2}>\pi s_{3}$ and $s_{2}>\pi s_{1}>\pi s_{3}$. The possibility relations induced by the plausibility orders $s_{1}$ $>\pi s_{3}>\pi s_{2}$ and $s_{2}>\pi s_{3}>\pi s_{1}$ also extend $>$ (and thus belong to $\boldsymbol{F}_{\boldsymbol{l}}(>)$ ) but are not refinements of $>_{\Pi *}$, even if they are more specific than it.

Finally, the next result proves that any semi-cancellative $>$ can be reconstructed from the linear possibility relations refining it.

Theorem 4. Let $>$ be the cautious substitute of some $S A R>_{L}$. Then $A>B$ if and only if $A>_{\Pi}$ $B$ for all linear possibility relations $\geq_{\Lambda}$ in $\boldsymbol{F}_{\boldsymbol{l}}(>)$.

Proof : It only remains to be proved that if $A>_{A} B$ for all linear possibility relations $\geq_{A}$ in $\boldsymbol{F}_{\boldsymbol{l}}(>)$, it implies $A>B$. For suppose $A>B$ is not true. Then it cannot hold that $B>A$. Otherwise, $B>{ }_{A} A$ for all linear possibility relations $\geq_{A}$ in

$\boldsymbol{F}_{\boldsymbol{l}}(>)$. Then neither $A>B$ nor $B>A$ holds. Then there exists a refinement $\geq_{\Pi}$ in $\boldsymbol{F}(>)$ such that $B$ $>_{\Pi} A$. To see it create a semi-cancellative $S A R>_{1}$ by enforcing $B>A$ in $>$. It does not create inconsistency, since $A>B$ is not supposed to hold. Choose $\geq_{\Pi}$ to be the least specific possibility relation induced by $>_{1}$. Then any linear possibilistic refinement $\geq_{\Lambda}$ of $>_{1}$ is in $\boldsymbol{F}_{\boldsymbol{l}}(>)$. It satisfies $B$ $>_{\Lambda} A$, which contradicts the assumption $A>_{\Lambda} B$ for all linear possibility relations $\geq_{\Lambda}$ in $\boldsymbol{F}_{\boldsymbol{l}}(>)$.

So any cautious substitute of a $S A R$ can be represented by a family of linear possibility relations. If $\boldsymbol{A}_{L}(C)$ denotes the set of accepted beliefs induced in the context $C$ by the acceptance relation $z_{L}$, and $A_{\Lambda}(C)$ the set of accepted beliefs induced by a linear possibility relation $\geq_{\Lambda}$ of $\boldsymbol{F}_{\boldsymbol{l}}$ $(>)$ where $>=\operatorname{cs}\left(>_{L}\right)$, then the above Theorem implies that :

$$
\boldsymbol{A}_{L}(C)=\cap \geq_{\Lambda} \in F_{l}(>) A_{\Lambda}(C)
$$

Note that $A_{\Lambda}(C)$ is a complete (maximal consistent) belief set, in the sense that $\forall A \subseteq C, A \in$ $A_{\Lambda}(C)$ or $C \backslash A \in A_{\Lambda}(C)$. Generally, more than one possibility relation is needed to represent $>$. However, sometimes, a semi-cancellative $S A R>$ is itself the strict part of a possibility relation. This is when $>$ is negatively transitive, so that its complement $\geq$ is transitive. Consider the complete relation $A \geq B$ if and only if $B>A$ does not hold. Clearly, $A \cup B \geq B$ and $A \cup B \geq A$ (otherwise, for instance, $A>A \cup B$, which implies $\varnothing>\varnothing$, and that is impossible). Due to proposition 6 , one of $A \geq A \cup B$ and $B \geq A \cup B$ is true. If $\geq$ is transitive, then it is clearly a possibility relation, and $\geq=\geq_{\pi^{*}}$.

\section{Applications to Related Areas}

The above results are not totally new at the mathematical level because they are closely related to former results in non-monotonic reasoning after (Kraus et al., 1990), and the AGM theory of belief revision. What is proposed here is rather a new understanding of the formal machinery already at work in these two already related areas. In the belief revision approach, the primitive 
concepts are a belief set often expressed in propositional logic, and the revision operation. However, the knowledge of the agent that produces this belief set and makes it evolve does not explicitly appear in the approach. In particular there is no clear distinction, inside the belief set, between observations received from outside by the agent, and beliefs produced by plausible inference. In the non-monotonic reasoning approach, the underlying knowledge is explicitly pointed out at the syntactic level under the form of a set of rules (or conditional assertions), closed under some inference process relying on basic postulates of plausible reasoning. Then in a given context, the belief set is formed by the set of conclusion parts of rules whose common condition part describes this context. In our approach we adopt a semantic view, considering the confidence relation as the primitive notion encoding the generic knowledge of an agent. Rules and belief sets are derived from the confidence relation, while the latter appear as a by-product of the theory in the other mentioned approaches. Our view also makes the analogy between plausible reasoning and probabilistic inference much clearer, although this connection has already been made in various ways.

\subsection{Confidence Relations and Non-Monotonic Reasoning}

The preferential approach of Kraus et al. (1990) to non-monotonic reasoning (also known as "system P") was described at the semantic level by Lehmann and Magidor (1992) in terms of rankings of states, by Fariñas del Cerro et al. (1994) and Dubois and Prade (1995a), in terms of families of possibility relations, and by Friedman and Halpern (1996) in a more general setting of partial orders of events, by means of so-called "plausibility measures" that are set-functions valued in a partially ordered set, basically what we call confidence relations. Following these works, it can be shown that the relation on disjoint events induced by a conditional knowledge base via system $\mathrm{P}$ is the disjoint graph of a $S A R$. Since this result is very close to the previous one, we present it only briefly here.

Let $A \rightarrow B$ be a conditional assertion relating two propositions. Again we neglect the syntactic side here and just write propositions as events. The rule $A \rightarrow B$ states that if $A$ holds then $B$ generally holds too. It should be understood as : in the context $A$, the agent accepts $B$. Kraus et al. (1990) discussed basic properties of non-monotonic inference :

$$
\begin{aligned}
& \text { Reflexivity }: A \rightarrow A \\
& \text { Right weakening }: A \rightarrow B \text { and } B \subseteq C \text { imply } A \rightarrow C \\
& \text { AND }: A \rightarrow B \text { and } A \rightarrow C \text { imply } A \rightarrow B \cap C \\
& \text { OR }: A \rightarrow C \text { and } B \rightarrow C \text { imply } A \cup B \rightarrow C \\
& \text { Cautious monotony }(C M): A \rightarrow B \text { and } A \rightarrow C \text { imply } A \cap B \rightarrow C \\
& \text { Cut }: A \rightarrow B \text { and } A \cap B \rightarrow C \text { imply } A \rightarrow C
\end{aligned}
$$

The above rules of inference embody the notion of plausible reasoning in the presence of incomplete information. Namely, they describe the properties of deduction under the assumption that the state of the world is as normal as can be. The crucial rules are Cautious Monotony and the Cut. Cautious Monotony claims that if $A$ holds, and if the normal course of things is that $B$ and $C$ hold in this situation, then knowing that $B$ and $A$ hold should not lead us to situations that are exceptional for $A: C$ should still normally hold. The Cut is the converse rule : if $C$ usually holds in the presence of $A$ and $B$ then, if situations where $A$ and $B$ hold are normal ones among those where $A$ holds (so that $A$ normally entails $B$ ), one should take it for granted that $A$ normally entails $C$ as well. The other properties are not specific to plausible inference : OR enables 


\section{REPRESENTATIONS OF ACCEPTANCE}

disjunctive information to be handled without resorting to cases. The Right Weakening rule, when combined with AND, just ensures that the set of non-monotonic consequences is deductively closed in every context. Reflexivity sounds natural but can be challenged for the contradiction $(A=\varnothing)$. These basic properties can be used to form the syntactic inference rules of a logic of plausible inference.

A strict order $>$ on disjoint events is induced by a set of conditional assertions $\Delta$ by interpreting a rule $A \rightarrow B$ as the statement that the joint event $A \cap B$ is more plausible than $A \cap \neg B$ :

$$
A \cap B>A \cap \neg B \text { iff } A \rightarrow B \in \Delta
$$

Equation (5) builds the disjoint graph of a confidence relation from a set of assertions. Conversely, starting from confidence relation $>_{L}$, a set of rules $\Delta$ is obtained as

$$
A \cup B \rightarrow \neg B \in \Delta \text { iff } A>_{L} B \text { for disjoint } A, B
$$

Equation (6) builds the set of conditional assertions corresponding to a disjoint graph. Using equation (5), the properties of non-monotonic preferential inference can be written as properties of the confidence order $>$. Especially :

$R W:$ If $B \subseteq C$ and $A \cap B>A \cap \neg B$ then $A \cap C>A \cap \neg C$

AND: If $A \cap B>A \cap \neg B$ and $A \cap C>A \cap \neg C$ then $A \cap B \cap C>A \cap(\neg B \cup \neg C)$

Conditional assertions induce strict acceptance relations and that it reciprocally turns out that the entailment relation induced by a strict acceptance relation is not more general than preferential inference, but for the reflexivity $A \rightarrow A$ when $A=\varnothing$. Namely the following result holds :

Proposition 10. If $\Delta$ is a conditional knowledge base closed under $R R, A N D, O R, R W, C M$, and $C P$, then the confidence relation it defines as $A \cap B>A \cap \neg B$ iff $A \rightarrow B \in \Delta$ is the disjoint graph of a SAR. Conversely, any SAR satisfies CCS, RW, CM, CUT, OR, CP, and $R R$. The corresponding family of conditional assertions satisfies system P but for $\varnothing \rightarrow \varnothing$.

Dubois et al. (2003) provide direct proofs. The AND and the RW axioms are exactly the CES and the CCS axioms of acceptance relations and the other properties of $\rightarrow$ can be proved from CCS, CES, QT, O and MI. Conversely, the main point is to prove that the KLM axioms enforce the negligibility and the transitivity properties of $>$.

Given a conditional knowledge base $\Delta=\left\{A_{i} \rightarrow B_{i}, i=1, n\right\}$, its preferential closure $\Delta^{P}$ is obtained by applying to it the KLM properties as inference rules. The above results clearly show that the relation on events induced by $\Delta^{P}$ is a SAR $>_{L}$, and that the set $\left\{A, C \rightarrow A \in \Delta^{P}\right\}$ coincides with the belief set $\boldsymbol{A}_{\mathrm{L}}(C)$. So, our characterization of acceptance relations (Definition 10) provides an axiomatics almost equivalent to system P. Something similar was done by Friedman and Halpern (1996) with their "plausibility" measures. The main difference between Friedman and Halpern's axiomatic and ours comes from a different understanding of system $\mathrm{P}$ on our side. Friedman and Halpern (1996) admit the reflexivity $\varnothing \rightarrow \varnothing$ of $\rightarrow$ and interpret $A \rightarrow B$ as $(A \cap B>$ $A \cap \neg B$ or $A=\varnothing$ ). Our simpler interpretation as $A \cap B>A \cap \neg B$ allows us to drop one axiom they 
need (namely, $A>B>\varnothing$ implies $A \cup B>\varnothing$ ), since it is vacuous here (SARs are non dogmatic). Actually, we never assume that the inference $A \rightarrow A$ holds when $A$ is empty. The non-dogmatic assumption $(A>\varnothing)$ provides for the reflexivity axiom when $A \neq \varnothing$.

The link between acceptance relations and System P also results from past works. Indeed, the relation defined by a consistent base of defaults can be represented by a non-empty family of possibility measures $\boldsymbol{F}_{\Delta}$ such that (Dubois \& Prade, 1995a; Benferhat et al., 1999a) $: A \rightarrow B \in \Delta$ if and only if $\forall>_{\Pi} \in \boldsymbol{F}_{\Delta}, A \cap B>_{\Pi} A \cap \neg B$. We have seen in section 2.5 that the relation $>$ defined in this way by means of a family of possibility relations is a SAR.

However, the reflexivity axiom of system $\mathrm{P}$ (i.e. $A \rightarrow A$ ) is hard to accept in the present framework since it means $A>\varnothing$ for all $A$, so we would get $\varnothing>\varnothing$ : it violates our irreflexivity requirement for $>$. But $A \rightarrow A$ makes sense for $A \neq \varnothing$, and we can consider a restricted reflexivity condition (Benferhat et al., 1997b) :

$$
R R: A \rightarrow A, \forall A \neq \varnothing,
$$

which writes : $A>\varnothing, \forall A \neq \varnothing$. Besides, $\varnothing>A$ never holds for a $S A R$. Hence the consistency preservation condition for $\varnothing$ :

$$
C P: \forall A, A \rightarrow \varnothing \text { never holds. }
$$

Axioms $\mathrm{CP}$ and $\mathrm{RR}$ should replace the reflexivity axiom $A \rightarrow A$, in the scope of acceptance relation semantics.

System $\mathrm{P}$ can be extended by adding the following axiom, originally due to Makinson:

$$
\text { Rational monotonicity : } \quad A \rightarrow C \text { and } \operatorname{not}(A \rightarrow \neg B) \text { imply } A \cap B \rightarrow C
$$

This is for instance the basis of the so-called "rational" inference of Lehmann (Lehmann \& Magidor, 1992). In the above, "not $(A \rightarrow \neg B)$ " means that it is not the case that $B$ generally does not hold, in situations where $A$ holds. Indeed if $\neg B$ is expected then it might well be an exceptional $A$-situation, where $C$ is no longer normal. In terms of confidence relations, this axiom reads:

RM : If $A \cap C>_{L} A \cap \neg C$, and $A \cap \neg B>_{L} A \cap B$ does not hold, then $A \cap B \cap C>_{L} A \cap B \cap \neg C$

It is well known that, if a property of rational monotony is added to the basic $K L M$ properties, then the non-monotonic inference $A \rightarrow B$ can always be modeled as $A \cap B>_{\Pi} A \cap \neg B$ for a possibility relation $\geq_{\Pi}$ (Benferhat et al., 1997b). So, when added to the axioms of system P, RM forces the confidence relation $\geq$ underlying a set of rules to be a complete preordering, hence a possibility relation. This result proves that any semi-cancellative SAR that satisfies RM is negatively transitive and reciprocally, that any negatively transitive semi-cancellative SAR satisfies RM.

A conditional knowledge base $\Delta$ is thus equivalent to a set of constraints of the form $A>B$ restricting a family of possibility relations. Benferhat et al. (1997b) showed that selecting the least specific possibility relation corresponds to the computation of the rational closure of $\Delta$ after Lehmann and Magidor (1992), or the most compact ranking according to Pearl (1990). The actual computation of this rational closure of $\Delta$ can be carried out by finding the wop induced by this set 


\section{REPRESENTATIONS OF ACCEPTANCE}

of constraints by means of a ranking algorithm, several versions of which have appeared in the literature (see e.g. the algorithm of system Z of Pearl(1990), or a similar algorithm of Benferhat et al. (1992) for possibilistic logic). The computation of a possibility relation from a semicancellative SAR (Section 3.2) by means of a ranking algorithm actually performs a similar closure computation.

\subsection{Revising accepted beliefs vs. revising an acceptance relation}

At this point, it can be asked whether, given a SAR $>_{L}$, the change operation, that turns the belief set $\boldsymbol{A}_{\mathrm{L}}$ into the belief set $\boldsymbol{A}_{L}(C)$ when the information stating that proposition $C$ is true, will satisfy the main postulates of belief revision (after Gärdenfors, 1988). A set of accepted beliefs is characterized by a subset of states $B_{L}{ }^{C}$ such that $A_{L}(C)=\left\{A, B_{L}{ }^{C} \subseteq A\right\} . B_{L}{ }^{C}$ is called the kernel of the belief set, and it is the set of states not excluded by the belief set. It is then possible to describe a belief change operation as a mapping from $2^{S} \times 2^{S}$ to $2^{S}$, changing a pair $(B, C)$ of subsets of states into another subset of states $B^{C}$. Suppose $B$ is the kernel of the belief set $K$ and $C$ is the input information. $B^{C}$ is the kernel of the revised belief set $K^{*} C$. This presentation is in some sense easier to understand than using the general logical setting used by Gärdenfors, which is a syntactic construction with a syntax-independence axiom. The translation of the postulates characterizing revision operations * then proceeds as follows :

Postulate 1: For any input $\mathrm{C}$, and any belief set $K, K^{*} C$ is a belief set. It means that for any pair of subsets $(B, C)$ of $S, B^{C}$ is a subset of $S$

Postulate $2: K^{*} C$ contains $C$. This is the success postulate, which reads $B^{C} \subseteq C$.

Postulate 3: $K^{*} C \subseteq \operatorname{Cons}(K \cup\{C\})$ where the latter is the belief set obtained by simply adding $C$ to $K$ and taking the logical closure (also called the expansion operation). In set-theoretic terms, it reads $B \cap C \subseteq B^{C}$.

Postulate 4: if $\neg C \notin K$ then $\operatorname{Cons}(K \cup\{C\}) \subseteq K^{*} C$. In other words, if the new information is coherent with the old belief set then it should be absorbed by it. In set-theoretic terms it reads : if $B \not \subset \neg C$, then $B^{C} \subseteq B \cap C$.

Postulate 5: If $C$ is a logical contradiction $(=\varnothing)$ then $K^{*} C$ contains all possible propositions. It means that $B^{\varnothing}=\varnothing$.

Postulate 6 is the claim for syntax invariance. It is obviously satisfied in the set-theoretic setting, and this is why the set-theoretic description of the revision theory makes sense.

Postulate 7: $K^{*}(B \cap D) \subseteq C o n s\left(K^{*} C \cup\{D\}\right)$. It means that $B^{C} \cap D \subseteq B^{C \cap D}$.

Postulate 8: If $\neg \phi \notin K^{*} \psi$ then $\operatorname{Cons}\left(K^{*} C \cup\{D\}\right) \subseteq K^{*}(B \cap D)$. It reads : if $B^{C} \not \subset \neg D$ then $B^{C \cap D} \subseteq$ $B^{C} \cap D$.

Postulates 1 and 2 are not valid for sets of accepted beliefs induced by an acceptance relation, because of the case $C=\varnothing$. Since $A_{\mathrm{L}}(\varnothing)$ is empty, $B^{\varnothing}$ simply does not exist. However these two postulates hold when restricting to non-contradictory input information $C \neq \varnothing$. Accordingly, 


\section{DUBOIS, FARGIER, \& PRADE}

Postulate 5 is not assumed in our framework. These restrictions make sense because the new information is supposed to be a piece of observed evidence on which the confidence relation will focus. It is impossible to focus on the empty set. Under the restriction of non-contradictory inputs (which makes postulate 5 vacuous), all postulates but two unsurprisingly hold in the acceptance relation setting. The fourth and the eighth ones are not valid (see Dubois et al. 2003). Requesting the validity of these two ones comes down to requesting that the acceptance relation be a possibility relation, as they are closely related to Rational Monotonicity.

The direct link between acceptance relations and revision postulates provided here is clearly reminiscent of the comparison between revision and non-monotonic inference, first studied by Makinson and Gärdenfors (1991). However, the postulates of acceptance are less numerous and easier to interpret than the postulates of belief revision. Moreover, our paper makes it clear that the AGM theory of revision is only concerned with the revision of the current beliefs of an agent pertaining to the present situation. It is not concerned with the revision of the generic knowledge of the agent regarding what is normal and what is not. The acceptance function setting is more general than the belief revision setting not only because less postulates are assumed, but because it lays bare the existence of two kinds of revision problems : the revision of accepted beliefs on the basis of new observations, and the revision of the acceptance relation itself, due to the arrival of new pieces of knowledge (such as a new default rule). For instance medical doctors generally do not modify their medical knowledge when getting new test results for a patient. They just revise their beliefs about the patient state. However, medical doctors may revise their medical knowledge when reading a specialized book or attending a medicine conference. The AGM theory deals with the first problem only and it corresponds to the notion of focusing the epistemic entrenchment relation on the proper reference class pointed at by the available factual evidence (see Dubois, Moral \& Prade, 1998).

The AGM revision theory only assumes that a belief set is replaced by another belief set, and it gives minimal rationality constraints relating the prior and the posterior belief sets. Thus doing, it may wrongly suggest that the posterior belief set depends on the prior one. The confidence relation framework shows that this is not the case. The calculation of the posterior belief set $\boldsymbol{A}_{L}(C)$ does not use the prior belief set. Both belief sets are built by means of plausible inference from generic knowledge encoded in the confidence relation. For the posterior belief set, the confidence relation is conditioned on the new context $C$ formed by all the available observations, including the new one. This point is not crystal-clear when adopting the notations of the AGM theory, where the epistemic entrenchment appears like a technical by-product of the formal construction.

Some people have wondered what becomes of the epistemic entrenchment relation after an AGM belief revision step. The acceptance relation framework makes it clear that it remains unchanged. Hence the claim that belief revision cannot be iterated, because the epistemic entrenchment relation that underlies the revision operation is lost when performing the next step, is questionable : if, after revising $\boldsymbol{A}_{\mathrm{L}}$ into $\boldsymbol{A}_{\mathrm{L}}(C)$, the agent receives a new reliable piece of information $D$ about the same static phenomenon, the belief set becomes $A_{\mathrm{L}}(C \cap D)$. Note that, by assumption, $C \curvearrowright D$ cannot be contradictory (otherwise one of $C$ or $D$ is wrong or they do not pertain to the same case). In the belief revision setting, it means that the following property holds: if $C \curvearrowright D \neq \varnothing,\left(K^{*} C\right)^{*} D=K^{*}(C \cap D)$. The same epistemic entrenchment should remain the same across successive revisions of contingent beliefs caused by new contingent non-conflicting observations. Similarly, in probabilistic reasoning (Pearl, 1988), the same Bayesian network is used when new observations come in. If the epistemic entrenchment must be revised, it means 


\section{REPRESENTATIONS OF ACCEPTANCE}

that the input information is a piece of generic knowledge, and such a kind of revision is not the purpose of the AGM theory; in the AGM terminology, this is like revising the revision operator (Lehmann, 1995).

The problem of revising the acceptance function is beyond the scope of this paper. Relevant research can be found in papers of Spohn (1988), Williams (1994), Boutilier and Goldszmidt (1995), Darwishe and Pearl (1997), and Benferhat et al. (1999b), although the distinction between the two types of revision is not always so clear from reading these works. Indeed, there is no consensus on a general and systematic approach to that kind of epistemic change in the literature, and the same can be observed for problems of revision of Bayesian networks (which pertain to probability kinematics, see Domotor, 1985).

\subsection{Plausible Inference versus Probabilistic Reasoning}

The originality of the confidence relation approach to plausible inference is that, instead of starting from intuitive postulates on syntactic objects (like Lehmann and colleagues), our basic concepts are on the one hand the confidence relation that is thought of as a natural tool for describing an agent's uncertain knowledge, and the notion of accepted belief on the other hand. This point of view enables plausible (non-monotonic) reasoning to be cast in the general framework of uncertain reasoning, which includes probabilistic reasoning. The analogy between non-monotonic inference and probabilistic reasoning has already been pointed out (Dubois \& Prade, 1994). It was stressed by Pearl (1988) and Lehmann and Magidor (1992) that the System P has semantics in terms of infinitesimal probabilities, and comes close to Adams (1975) conditional logic. Paris (1994) has viewed maximum entropy inference as a kind of default probabilistic inference. The selection of a most cautious comparative possibility relation in agreement with an acceptance relation is similar to the selection of a unique probability measure using maximal entropy (Maung, 1995). The relationship between maxent probabilistic reasoning and non-monotonic inference is further explored by Kern-Isberner (2001). Biazzo et al., (2002) reinterpret System $\mathrm{P}$ in the light of probabilistic logic under coherence in the De Finetti style. They show that, under this view, a conditional assertion $A \rightarrow B$ can be simply expressed by $P(B / A)=1$.

In probabilistic reasoning, the confidence relation stems from a probability measure or a family thereof. A set of generic rules is then encoded as a set of conditional probabilities $\mathrm{c}$ characterizing a family of probability measures (Paris, 1994). The most popular approach in AI currently uses a single probability measure, and the set of conditional probabilities defines a Bayesian network (Pearl, 1988). A Bayesian network really represents generic knowledge, like any confidence relation. This network is built either from expert domain knowledge, or from statistical data via learning techniques. Probabilistic inference with a Bayesian network consists in calculating the (statistical) conditional probability of a conclusion, where the conditioning event encodes the available observations (Pearl, 1988). The obtained conditional probability value is interpreted as the degree of belief of the conclusion in the current situation, assuming that this situation is a regular one in the context described by the observations. This procedure is very similar to the derivation of a plausible conclusion by conditioning an acceptance relation, or by deducing a rule from a rule base. The derived rule is valid "generally". Its conclusion is considered as an accepted belief in the current situation assuming that this situation is not an exceptional one in the context described by the observations modeled by the condition part of the derived rule. There is in fact a strong similarity between conditional probability and conditional possibility, and an ordinal form

of Bayes rule exists for possibility theory (Dubois \& Prade, 1998). Boolean conditional 
acceptance functions $A c$ of the form $A c(A \cap C)=1$ iff $A \cap C>_{L} \neg A \cap C$, and 0 otherwise have been used by Ben Amor et al (2002) in the investigation of qualitative counterparts of probabilistic independence.

Of course, there are also noticeable differences between probabilistic reasoning and ordinal plausible inference :

i) Plausible inference does not quantify belief, probabilistic inference does;

ii) Plausible reasoning considers the most plausible situations and neglects others, while probability theory performs reasoning in the average.

iii) Lastly, probabilistic reasoning is often not compatible with the notion of accepted belief. Indeed, the conjunction of two highly probable events may fail to be highly probable and may even turn out to be very improbable. However, the arbitrary conjunction of accepted beliefs is still an accepted belief (this is because we assume that the agent considers accepted beliefs as tentatively true). This is the source of the lottery paradox described in Section 2.1.

The solution to the lottery paradox lies in the existence of probability functions that agree with classical deduction from accepted beliefs. Such probabilities exist and have been laid bare in the previous section : big-stepped (or lexicographic) probabilities on finite sets. These very special probability functions actually provide a standard probabilistic semantics to system P (Benferhat et al., 1999a). Note that in the lottery paradox, it is implicitly assumed that all players have equal chance of winning. The underlying probability measure is uniform. Hence there is no regularity at all in this game : no particular occurrence is typical and randomness prevails. It is thus unlikely that an agent can come up with a consistent set of default rules about the lottery game. So, in the situation described in the counterexample, deriving accepted beliefs and reasoning from them is not advisable indeed.

However, big-stepped probabilities are the total opposite of uniformly distributed ones, since the probability of any state is larger than the sum of the probabilities of less probable states. So, the paradox disappears if the underlying phenomenon on which the agent entertains accepted beliefs is ruled by a big-stepped probability since $\{A, P(A / C)>0.5\}$ remains logically consistent and deductively closed. It suggests that big-stepped probabilities and plausible reasoning based on acceptance relations or system $\mathrm{P}$ model an agent reasoning in front of phenomena that have typical features, where some non-trivial events are undoubtedly more frequent than other ones. We suggest that such domains, where a body of default knowledge exists, can be statistically modeled by big-stepped probabilities on a suitable partition of the sample space. Default reasoning should then be restricted to such situations in order to escape the lottery paradox. It is always possible to build a big-stepped probability from a large state space $S$. One technique is to find the smallest set $E_{1}$ such that $P\left(E_{l}\right)>P\left(\neg E_{l}\right)$. Then, the same procedure is applied changing $S$ into $\neg E_{1}$, etc. Clearly, in the case of a uniform distribution on $\mathrm{S}$, the set $E_{1}$ is not unique and its choice is very arbitrary. So would be the so-obtained big-stepped probability. On the contrary, if the distribution on $\mathrm{S}$ is far from being uniform, the whole big-stepped probability is likely to be unique and set $E_{l}$ will cluster the most frequent states.

While we cannot expect to find sample spaces displaying such kinds of statistical big-stepped probability functions right away, one may think that for phenomena that have regularities, there may exist partitions of the sample space that form conceptually meaningful clusters of states for the agent and that can be ordered via a big -stepped probability. If this conjecture is valid, it points out a potential link between non-monotonic reasoning and statistical data, in a knowledge discovery perspective. An interesting problem along this line is as follows : Given statistical data on a sample space and a language, find the "best" linguistically meaningful partition(s) of the 


\section{REPRESENTATIONS OF ACCEPTANCE}

sample space, on which big-stepped probabilities are induced and default rules can be extracted (see Benferhat et al., 2002, for preliminary results). The difference between other rule extraction techniques and the one suggested here, is that, in our view, the presence of exceptions is acknowledged in the very definition of symbolic rules, without having to keep track of the proportion of such exceptions.

\section{Summary and Conclusions}

This paper can be seen as an exploration of the common ground between classical deductive reasoning, non-monotonic reasoning and probabilistic reasoning. By considering general orderings between events that model the concept of relative strength of belief, and moreover enforcing the notion of deductive closure, it is proved that the corresponding confidence orderings can always be modeled by families of possibility relations. It confirms the idea that possibility theory is the uncertainty theory that is the most compatible with classical logic. This is already suggested by the fact that possibilistic logic (Dubois et al., 1994) is a much simpler extension of propositional logic than probabilistic logic. Possibilistic logic can encode nonmonotonic reasoning in the style of Lehmann and colleagues.

Our results provide a wide perspective encompassing probabilistic reasoning, possibility theory, belief revision, and non-monotonic reasoning, from a rather simple and general starting point : accepted beliefs induced by confidence relations. It casts some light on the AGM belief revision theory, by showing the difference between revising a set of accepted beliefs upon the arrival of some observed fact, and revising a confidence relation due to the acquisition of some generic knowledge. The first kind of revision is captured by conditioning the confidence relation on the proper reference class, in full harmony with probabilistic reasoning. The other type of revision, which alters the normality ordering of states, is a different problem, not addressed here.

Our results are quite negative regarding the general compatibility between logical representation of what logic-based AI calls beliefs, viewed here as accepted propositions, for which deductive closure is allowed, and partial belief in the sense of probability theory. These results are a severe impediment to a generalized view of theory revision based on orders on formulae derived from uncertainty theories other than epistemic entrenchments, simply because the assumption of closed belief sets is devastating in this respect.

The paper seems to confirm that the notion of deductive closure is not fully adapted to the modeling of belief, not only because this notion disregards syntactic aspects and presupposes logical omniscience, but because the closure under conjunction may be counter-intuitive when reasoning with partial belief, as already revealed in the lottery paradox. Our solution to this paradox is to consider that non-monotonic reasoning with accepted beliefs makes sense in situations where trends of normality can be clearly discerned : some states of nature are so much frequent that they are considered normal, expected. This is obviously the case in everyday life (planes usually do not crash, houses usually do not collapse, etc.) The lottery paradox makes more sense for situations where random phenomena prevail (like in games of chance).

In order to model symbolic reasoning methods that come closer to uncertain reasoning with partial beliefs, weaker types of "deductive closures" might be considered for this purpose, as for instance unions of standard deductively closed sets of propositions (that may be globally inconsistent). This type of closure is encountered in argument-based reasoning under inconsistency (Benferhat et al., 1997). Tolerating inconsistency is indeed incompatible with standard deductive closure. It turns out that most confidence functions (and noticeably probabilities) synthesize partially conflicting pieces of information while possibility measures do 
not (as witnessed by their nested structure). It may explain why possibility theory seems to be the only simple uncertainty calculus that accounts for the concept of accepted beliefs in the sense of propositions one can reason about as if they were true.

Finally, further work is needed to lay bare numerical uncertainty functions other than probabilities and possibilities that induce acceptance relations. In Dempster-Shafer theory (Shafer, 1976), preliminary results (Dubois \& Prade, 1995a; Dubois, Fargier and Prade, 1998) suggest that many plausibility functions that combine possibility measures and lexicographic probabilities are compatible with acceptance postulates.

\section{Appendix A. Relationships Between Axioms O and MI}

Proposition A. 1 If $\geq_{L}$ satisfies MI and T, then $A>_{L} B$ and $B \geq_{L} C$ imply $A>_{L} C$.

Proof: It is obvious that $A>_{L} B$ and $B \geq_{L} C$ imply $A \geq_{L} C$, due to $T$. If $C>_{L} A$, then due to $B \geq_{L} C$, $T$ again implies $B \geq_{L} A$, which contradicts $A>_{L} B$. So, $A>_{L} C$.

Corollary: If $\geq_{L}$ satisfies $M I$ and $T$, then it satisfies $O$.

Proof: By MI, $A \subseteq A^{\prime}$ implies $A^{\prime} \geq_{L} A$ and $B^{\prime} \subseteq B$ implies $B \geq_{L} B^{\prime}$. The previous property allows to deduce $A^{\prime}>_{L} B$ from $A>_{L} B$ and $A^{\prime} \geq_{L} A$ and $A^{\prime}>_{L} B^{\prime}$ from $A^{\prime}>_{L} B$ and $B \geq_{L} B^{\prime}$.

Proposition A.2 If $\geq_{L}$ is complete and satisfies $O$, then it satisfies $M I$.

Proof: Let $A, B$ be such that $A \subseteq B$. By completeness, $A \geq_{L} A$. By O:we get $A \cup(B \backslash A) \geq_{L} A$, i.e., $B \geq_{L} A$.

\section{References}

Adams E.W. (1975). The Logic of Conditionals. Dordrecht : D. Reidel

Alchourrón C.E.P., Gärdenfors P., \& Makinson D. (1985). On the logic of theory change : Partial meet functions for contraction and revision. J. of Symbolic Logic, 50, 513-530.

Bellman R., Cooke R.L., \& Lockett J. (1970). Algorithms, Graphs and Computers., New-York, N.Y. : Academic Press.

Ben Amor N., Mellouli K., Benferhat S., Dubois D., \& Prade H. (2002). A theoretical framework for possibilistic independence in a weakly ordered setting. Int. J. of Uncertainty, Fuzziness and Knowledge-Based Systems, 10, 117-155.

Benferhat S., Dubois D., \& Prade H. (1992). Representing default rules in possibilistic logic. Proc. of the 3rd Inter. Conf. on Principles of Knowledge Representation and Reasoning (KR'92)., Cambridge, MA, 673-684.

Benferhat S., Dubois D., \& Prade H. (1997a). Some syntactic approaches to the handling of inconsistent knowledge bases : a comparative study. Studia Logica, 58, 17-45.

Benferhat S., Dubois D., \& Prade H. (1997b). Non-monotonic reasoning, conditional objects and possibility theory. Artificial Intelligence, 92, 259-276. 


\section{REPRESENTATIONS OF ACCEPTANCE}

Benferhat S., Dubois D., \& Prade H. (1999a). Possibilistic and standard probabilistic semantics of conditional knowledge. Journal of Logic and Computation, 9, 873-895, 1999.

Benferhat S., Dubois D., \& Papini O. (1999b). A sequential reversible belief revision method based on polynomials. Proceedings National American AI conference (AAAI-99), Orlando, Floride (USA) AAAI Press/The MIT Press, p. 733-738.

Benferhat S., Dubois D., Lagrue S., \& Prade H. (2002). A big-stepped probability approach for discovering default rules. Proc. of the Ninth International Conference, Information Processing and Management of Uncertainty in Knowledge-based Systems (IPMU 2002), Annecy, France, p. 283-289.

Biazzo V., Gilio A., Lukasiewicz T., \& Sanfilippo G. Probabilistic Logic under Coherence, Model Theoretic Probabilistic Logic, and Default Reasoning in System P. J. Applied NonClassical Logics, 12(2), 2002.

Boutilier C., \& Goldszmidt M. (1995). Revision by conditionals beliefs. In G. Crocco et al., (Eds.): Conditionals : From Philosophy to Computer Sciences. Oxford, UK: Oxford University Press.

Choquet G. (1953). Theory of capacities, Ann. Inst. Fourier (Grenoble), 5, 131-295.

Cohen L. J. (1977). The Probable and the Provable. Oxford, U.K. : Clarendon Press.

Cohen L. J. (1989). Belief and acceptance. Mind, XCVIII, No. 391, 367-390.

Cox R.T. (1961). The algebra of probable inference. The John Hopkins Press, Baltimore.

Darwishe A., \& Pearl J. (1997). On the logic of iterated belief revision. Artificial Intelligence, 89, $1-29$.

De Finetti B. (1974). Theory of Probability, New York : Wiley.

Domotor Z. (1985). Probability kinematics - Conditional and entropy principles. Synthese, 63, 74-115.

Dubois D. (1986). Belief structures, possibility theory and decomposable confidence measures on finite sets. Computers and Artificial Intelligence (Bratislava), 5(5), 403-416.

Dubois D., Fargier H., \& Prade H. (1997). Decision making under ordinal preferences and comparative uncertainty. Proc. of the $13 \mathrm{~h}$ Conf. on Uncertainty in Artificial Intelligence, Providence, RI, 157-164.

Dubois D., Fargier H., \& Prade H. (1998). Comparative uncertainty, belief functions and accepted beliefs. Proc. of 14th Conf. on Uncertainty in Artificial Intelligence, Madison, Wi., 113120

Dubois D., Fargier H., \& Prade H. (2003). Acceptance, Conditionals, and Belief Revision. Proc. of the Workshop on Conditionals, Information and Inference, Hagen, May 2002, to appear in Lecture Notes in AI series.

Dubois D., Lang J., \& Prade H. (1994). Possibilistic logic. In D.M. Gabbay et al. (Eds.) Handbook of Logic in Artificial Intelligence and Logic Programming, Oxford, UK: Oxford University Press, 439-513 


\section{DUBOIS, FARGIER, \& PRADE}

Dubois D., Moral S., \& Prade H. (1998). Belief change rules in ordinal and numerical uncertainty theories. in D. Dubois \& H. Prade, (Eds.) : Belief Change, Handbook on Defeasible Reasoning and Uncertainty Management Systems, Vol. 3. Dordrecht, The Netherlands : Kluwer Academic Publ., 311-392.

Dubois D., \& Prade H. (1991). Epistemic entrenchment and possibilistic logic. Artificial Intelligence 50, 223-239.

Dubois D., \& Prade H. (1992). Belief change and possibility theory. In P. Gärdenfors, (Ed.), Belief Revision, Cambriidge, U.K. : Cambridge University Press, 142-182.

Dubois D., \& Prade H. (1994). Non-standard theories of uncertainty in knowledge representation and reasoning. The Knowledge Engineering Review, 9(4), 399-416.

Dubois D., \& Prade H. (1995a). Conditional objects, possibility theory and default rules. In G. Crocco et al., (Eds.) : Conditionals : From Philosophy to Computer Sciences Oxford, UK : Oxford University Press, 311-346.

Dubois D., \& Prade H. (1995b), Numerical representation of acceptance. Proc. of the 11th Conf. on Uncertainty in Artificial Intelligence, Montréal, 149-156.

Dubois D., \& Prade H. (1998). Possibility theory : qualitative and quantitative aspects. In P. Smets (Ed.), Quantified Representation of Uncertainty and Imprecision, Handbook of Defeasible Reasoning and Uncertainty Management Systems, Vol. 1. Dordrecht, The Netherlands : Kluwer Academic Publ., 169-226

Fariñas del Cerro L., \& Herzig A. (1991). A modal analysis of possibility theory. Proc. of the Inter. Workshop on Fundamentals of Artificial Intelligence Research Smolenice Castle, Czechoslovakia, Lecture Notes in Computer Sciences, Vol. 535. Berlin : Springer Verlag, 11-18.

Fariñas del Cerro L., Herzig A.. \& Lang J. (1994). From ordering-based non-monotonic reasoning to conditional logics. Artificial Intelligence, 66, 375-393.

Fishburn P. (1986). The axioms of subjective probabilities. Statistical Science, 1, 335-358.

Friedman N., Halpern J. (1996). Plausibility measures and default reasoning. Proc of the 13th National Conf. on Artificial Intelligence, Portland, OR, 1297-1304.

Gärdenfors P. (1988). Knowledge in flux. Cambridge, MA : MIT press.

Grove A. (1988). Two modellings for theory change. J. Philos. Logic, 17, 157-170.

Halpern J. (1997). Defining relative likelihood in partially-ordered preferential structures. $J$. $A I$ Research, 7, 1-24

Halpern J. (1999). Cox's Theorem revisited Journal of AI Research 11, 1999, pp. 429-435.

Kern-Isberner G. (2001). Conditionals in Non-monotonic Reasoning and Belief Revision. Lecture Notes in Artificial Intelligence, Vol. 2087, Berlin : Springer Verlag.

Kraft C.H., Pratt J.W \& Seidenberg A. (1959). Intuitive probability on finite sets. Ann. Math. Stat. 30, 408-419. 


\section{REPRESENTATIONS OF ACCEPTANCE}

Kraus S., Lehmann D., \& Magidor M. (1990). Non-monotonic reasoning, preferential models and cumulative logics. Artificial Intelligence, 44, 167-207.

Kyburg H. E. (1961). Probability and the Logic of Rational Belief. Wesleyan University Press. Middletown, $\mathrm{Ct}$.

Kyburg H.E. (1970). Conjunctivitis. In Swain (1970), op. cit., 55-82.

Kyburg H.E. (1988). Knowledge. In J. F. Lemmer and L. N. Kanal (Eds): Uncertainty in Artificial Intelligence, vol 2, Amsterdam : Elsevier, 263-272.

Lehmann D. (1995). Belief revision, revised. Proc. of the 14th Inter. Joint Conf. on Artificial Intelligence, Montreal, Quebec, 1534-1540.

Lehmann D. (1996). Generalized qualitative probability : Savage revisited. Proc. of the 12th Conf. on Uncertainty in Artificial Intelligence, Portland, 381-388.

Lehmann D., \& Magidor M. (1992). What does a conditional knowledge base entail? Artificial Intelligence, 55(1), 1-60.

Lewis D. L. (1973). Counterfactuals. Oxford, UK : Basil Blackwell.

Makinson D. \& Gärdenfors P. (1991). Relations between the logic of theory change and nonmonotonic reasoning. In A. Fürmann, M. Morreau, (Eds) : The Logic of Theory Change, LNAI 465, Berlin : Springer Verlag, 185-205.

Maung I. (1995). Two characterizations of a minimum information principle for possibilistic reasoning, Int. J. Approximate Reasoning, 12, 133-156.

Minker J., Ed., (2000). Logic-based Artificial Intelligence. Boston: Kluwer Academic Publishers.

Paris J. (1994). The Uncertain Reasoner's Companion. Cambridge, UK : Cambridge University Press.

Pearl J. (1988). Probabilistic Reasoning Intelligent Systems : Networks of Plausible Inference. San Mateo, CA, Morgan Kaufmann.

Pearl J. (1990). System Z: a natural ordering of defaults with tractable applications to default reasoning. Proc. of the 3rd Conf. on the Theoretical Aspects of Reasoning About Knowledge, San Mateo, CA, Morgan Kaufmann, 121-135.

Poole D. (1991). The effect of knowledge on belief : conditioning, specificity and the lottery paradox in defaut reasoning. Artificial Intelligence, 49, 281-307

Roth H. (2001). Change, Choice and Inference : A Study of Belief Revision and Non-monotonic Reasoning, Oxford Logic Guides, Bd. 42, Oxford, U.K : Oxford University Press.

Roubens M., Vincke P. (1985). Preference Modelling. Lecture Notes in Economics and Mathematical Systems, Vol. 250, Berlin : Springer Verlag.

Shafer G. (1976). A Mathematical Theory of Evidence. Princeton, NJ : Princeton University Press.

Shoham Y. (1988). Reasoning About Change - Time and Causation from the Standpoint of Artificial Intelligence, Cambridge, MA : The MIT Press. 
Snow P. (1999). Diverse confidence levels in a probabilistic semantics for conditional logics. Artificial Intelligence 113, 269-279.

Spohn W. (1988). Ordinal conditional functions : A dynamic theory of epistemic states. In W.L. Harper, B. Skyrms, (Eds.) : Causation in Decision, Belief Change, and Statistics, Vol. 2., Dordrecht : D. Reidel, 105-134.

Sugeno M. (1974). Theory of fuzzy integrals and its applications, Ph. D. Thesis, Tokyo Institute of Technology, Japan.

Swain M., Ed. (1970). Induction, Acceptance and Rational Belief, Dordrecht : D. Reidel.

Williams M.A. (1994). Transmutations of knowledge systems. Proc. of the 4th Inter. Conf. on Principles of Knowledge Representation and Reasoning, San Mateo, CA, Morgan Kaufmann, 619-629.

Wong S. K. M., Yao Y. Y., Bollmann P., Burger H. C. (1991). Axiomatization of qualitative belief structure. IEEE Transactions on Systems, Man \& Cybernetics, vol 21 no 4, 726-734.

Zadeh L. A. (1978). Fuzzy sets as a basis for a possibility theory, Fuzzy Sets and Systems, 1, 3-28. 